\title{
A persistent behavioral state enables sustained predation of humans by mosquitoes
}

\author{
Trevor R. Sorrells ${ }^{1,2}$, Anjali Pandey ${ }^{1}$, Adriana Rosas-Villegas ${ }^{1}$, Leslie B. Vosshall1-3
}

\section{SUMMARY}

\begin{abstract}
Predatory animals first detect, then pursue, and ultimately capture prey. Sensory cues, including scent emitted by prey, are detected by the predator and used to guide pursuit. Because the pursuit phase can last for extended periods of time, it is critical for predators to persist in the chase even when prey is difficult to detect in a noisy sensory landscape. It is equally important for predators to abandon pursuit if enough time has elapsed that prey capture is unlikely to occur. We studied prey detection and sustained pursuit in the mosquito Aedes aegypti, a micropredator of humans. These animals first detect humans through sensory cues that are emitted at a distance such as carbon dioxide in breath and odor from skin. As the mosquito approaches a human, additional cues such as body heat and visual contrast signal the promise of a blood meal, which females need to produce eggs. To study how initial prey detection influences the duration of pursuit, we developed optogenetic tools to induce a brief fictive sensation of carbon dioxide and used machine learning-based classification of behavior to investigate how mosquitoes respond to subsequent human cues. We found that a 5-second optogenetic pulse of fictive carbon dioxide induced a persistent behavioral state in female mosquitoes that lasted for more than 10 minutes. This state is highly specific to females searching for a blood meal and was not induced in recently blood-fed females or in males, who do not feed on blood. In males that lack the gene fruitless, which controls persistent social behaviors in other insects, fictive carbon dioxide induced a long-lasting behavior response resembling the predatory state of females. Finally, we show that the persistent state triggered by detection of fictive carbon dioxide enabled females to engorge on a blood meal mimic offered up to 14 minutes after the initial stimulus. Our results demonstrate that a persistent internal state allows female mosquitoes to integrate multiple human sensory cues over long timescales, an ability that is key to their success as an apex micropredator of humans.
\end{abstract}

\section{INTRODUCTION}

Predatory animals employ varied strategies to locate and capture their prey. Ambush predators wait for prey to come into close proximity, whereas pursuit predators travel long distances, stalking or chasing their prey. All predatory strategies for capturing prey must balance the need to obtain food with the expenditure of energy on unsuccessful hunts $(1,2)$. The duration of a pursuit could depend on the predator continuously sensing prey stimuli. Alternatively, it may be sustained by short-term memory or a change in the internal state of the predator that outlasts individual prey stimuli.

Ambush predators are opportunists that wait for prey to approach them, in some cases creating hidden nests or traps or luring their prey with an attractant. Among blood-feeding arthropods, ticks exclusively ambush their hosts, climbing to the top of plants and attaching onto passing animals and humans (3). In contrast, pursuit predators use sensory stimuli to detect, stalk, and capture prey over longer time periods. Pursuit predators can rely predominantly on visual or auditory tracking while others employ a more multisensory approach $(4,5)$. Sharks use olfactory cues over long distances, then at closer ranges rely on vision, electroreception, and hydrodynamic imaging $(6,7)$. The early stalking phase of pursuit predators is important to the success of the hunt (2). During this phase the predator may receive sensory information intermittently, for instance losing sight of their prey, while continuing to stalk. Little is known about how predators retain the internal motivation to pursue prey over the course of the hunt.

\footnotetext{
${ }^{1}$ Laboratory of Neurogenetics and Behavior, The Rockefeller University, New York, NY 10065 USA, ${ }^{2}$ Kavli Neural Systems Institute, New York, NY 10065 USA, ${ }^{3}$ Howard Hughes Medical Institute, New York, NY 10065 USA. Current address: Department of Biology, Brandeis University, 415 South Street, Waltham, MA 02453 United States (A.P.) Correspondence: trevorsorrells@gmail.com (T.R.S.), leslie.vosshall@rockefeller.edu (L.B.V.)
} 
Micropredators such as the mosquito consume small quantities of their live prey rather than killing them outright (8), but employ similar tactics to other pursuit predators. Female mosquitoes combine rich multisensory information from olfactory, visual, taste, mechanosensory, and contact chemosensory systems to hunt humans from whom they obtain blood to produce eggs. Carbon dioxide $\left(\mathrm{CO}_{2}\right)$ produced by human breath is highly volatile, traveling long distances from the human host. Detection of $\mathrm{CO}_{2}$ by the mosquito results in an increase in flying behavior $(9,10)$ and upwind flight (11-13) that is often referred to as "activation." However, $\mathrm{CO}_{2}$ alone is not sufficient for short-range attraction or to elicit blood feeding. Mosquitoes require an additional, more proximal host cue such as body heat or skin odor for short-range attraction and to engorge on blood (10-15) (Figure $1 \mathrm{~A}$ ). In natural settings, human sensory cues are typically brief and intermittent by the time they reach the mosquito due to turbulent air flows and long distances (16). After initial detection of the host, mosquitoes must decide how long to pursue before giving up if they fail to detect additional sensory cues. If mosquitoes possess the ability to retain information about their prey and combine it with future information this may explain their success at locating and feeding on human blood. This idea has not previously been tested experimentally.

To study pursuit predation in the mosquito, we developed optogenetic tools to precisely deliver short pulses of fictive $\mathrm{CO}_{2}$. This allowed us to test the effect of activating $\mathrm{CO}_{2}$ sensory neurons with greater temporal resolution and without the continuous stimulus of air flow required for delivery of real $\mathrm{CO}_{2}$. We observed that detection of fictive prey led to a long-lasting behavioral change in female mosquitoes. Following a 5-second fictive $\mathrm{CO}_{2}$ stimulus, animals exhibited high-arousal behaviors and engorged on a blood meal mimic offered up to 14 minutes later. Neither males nor previously blood-fed females showed these effects, and this persistent internal state was not induced by optogenetic stimulation of a sweet taste pathway. Remarkably, males lacking the fruitless gene showed long-lasting responses to fictive $\mathrm{CO}_{2}$ resembling those in females, consistent with our prior observation that these mutants show some aspects of female mosquito behavior (17). Our work identifies a persistent internal state that may explain how mosquitoes aggressively pursue human hosts for many minutes.

\section{RESULTS}

Fictive $\mathrm{CO}_{2}$ triggers blood feeding

We created optogenetic tools in Aedes aegypti mosquitoes that allowed us to precisely activate sensory neurons that are specialized to detect $\mathrm{CO}_{2}$. To do this we generated a transgenic strain that expresses the red light-activated cation channel CsChrimson translationally fused to the tdTomato fluorescent reporter (18). This was placed under control of the QF2/QUAS bipartite transcription system (19). We crossed this transgene into a strain that expresses the QF2 transcription factor in neurons that express the $\mathrm{Gr} 3 \mathrm{CO}_{2}$ receptor subunit $(10,20)$ (Figure 1B). CsChrimsontdTomato was detected in maxillary palp neurons but not antennal neurons, consistent with the observation that maxillary palp neurons are exquisitely sensitive to $\mathrm{CO}_{2}(21)$ (Figure $1 C, D)$. As expected, we found that CsChrimson-expressing neurons extended axons that innervated glomerulus MD1 in the antennal lobe of the mosquito brain (Figure $1 \mathrm{E})$, which is known to be $\mathrm{CO}_{2}$-sensitive (20). To test whether mosquitoes responded to optogenetic activation of $\mathrm{CO}_{2}$ sensory neurons, we presented animals with a 5-second red light $(627 \mathrm{~nm})$ stimulus and tracked their movement (Figure $1 \mathrm{~F}-\mathrm{I}$ ). Control animals carrying only the Gr3-QF2 driver or the QUAS-CsChrimson transgene reporter showed no response to red light. However, mosquitoes with both genetic elements ( $\mathrm{Gr} 3>\mathrm{CsChrimson)}$ increased their velocity in response to the stimulus (Figure $1 \mathrm{H}-\mathrm{I}$ ). This is consistent with the known role of $\mathrm{CO}_{2}$ in activating mosquitoes.

When combined with another host cue such as heat, $\mathrm{CO}_{2}$ is sufficient to elicit blood feeding in female mosquitoes (10). To test whether fictive $\mathrm{CO}_{2}$ sensation triggered by optogenetic activation of Gr3 sensory neurons could replace real $\mathrm{CO}_{2}$, we created a behavior assay called the opto-membrane feeder (Figure 1J). This assay consists of a cylindrical canister of mosquitoes surrounded by red light-emitting diodes (LEDs) and a warm blood meal behind a membrane sitting on top of the mesh lid. Mosquitoes were presented with either $\mathrm{CO}_{2}$, fictive $\mathrm{CO}_{2}$ via red light, or neither stimulus. Control mosquitoes with either Gr3-QF2 or QUASCsChrimson were attracted to the warm blood meal and engorged only when presented with $\mathrm{CO}_{2}$ but not when presented with red light (Figure 1K-M). In contrast, Gr3 > CsChrimson mosquitoes were attracted to the heated surface of the feeder and engorged in the presence of either real or fictive $\mathrm{CO}_{2}$, the latter delivered as a red-light stimulus (Figure 1K-M). These results demonstrate that mosquitoes interpreted optogenetic activation of the $\mathrm{CO}_{2}$ sensory neurons as a host cue that is sufficient to drive blood feeding.

Fictive $\mathrm{CO}_{2}$ induces prolonged host-seeking behaviors Host-seeking begins when female mosquitoes detect a human, typically by sensing volatile cues like $\mathrm{CO}_{2}$. Once activated by human odorants, they seek out the source of the cues, and upon landing, mosquitoes walk to locate a patch of skin to pierce. The behaviors mosquitoes exhibit and their duration could indicate what information is contained within a brief host stimulus and how long that information is relevant. To understand the timing and nature of the mosquito response to transient host cues, we created an assay called the opto-thermocycler (Figure 2A-B). In this assay mosquitoes receive optogenetic light stimulation from above and heat through the mesh at the bottom of the assay chamber. We employed machine-learning based behavior classification as a high-throughput readout of mosquito behavioral responses (Figure 2C-D). We tracked nine points on the mosquito body using Animal Part Tracker and four behaviors using JAABA (22): grooming, flying, walking, and probing, a behavior in which the mosquito inserts its proboscis through the mesh in the bottom of the container. All classifiers showed high accuracy with $>90 \%$ true positive and true negative rates on a set of test video frames (Data File S1). 
bioRxiv preprint doi: https://doi.org/10.1101/2021.10.06.463436; this version posted October 7, 2021. The copyright holder for this preprint (which was not certified by peer review) is the author/funder, who has granted bioRxiv a license to display the preprint in perpetuity. It is made available under aCC-BY 4.0 International license.

A
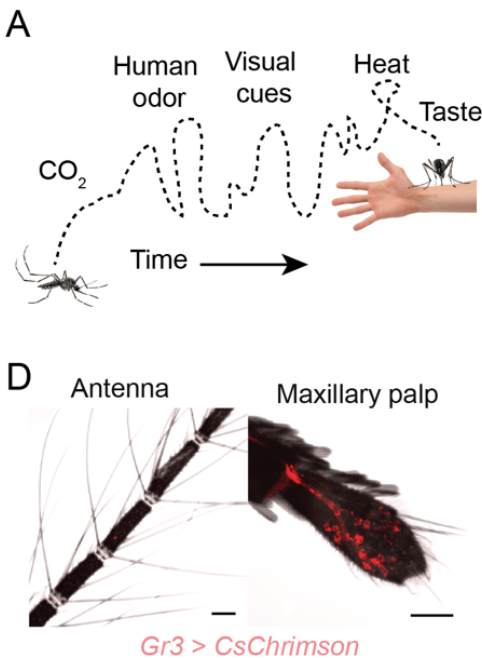

B

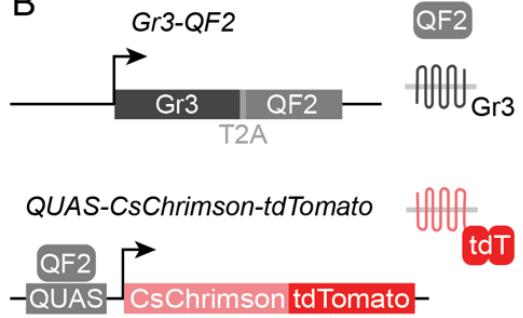

E
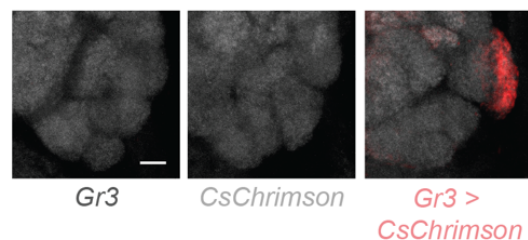

C

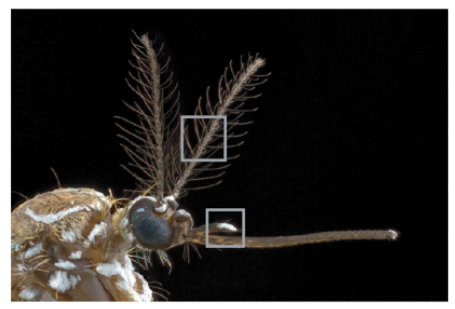

F $\quad$ Camera $627 \mathrm{~nm}$ light

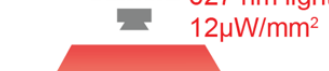

BRP tdTomato

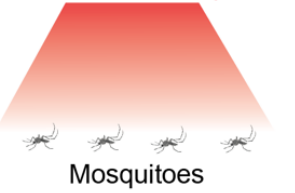

Mosquitoes
G

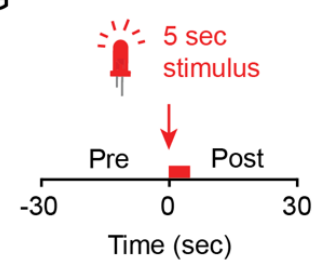

$J$
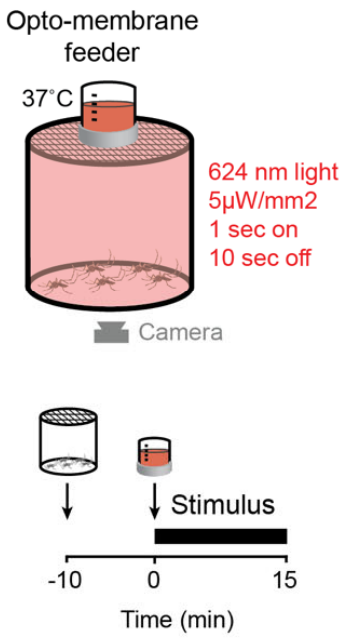

$-\mathrm{CO}_{2}$
$-\quad{ }^{\prime \prime}$ Light
$-\quad$ No stimulus
$\mathrm{H}$
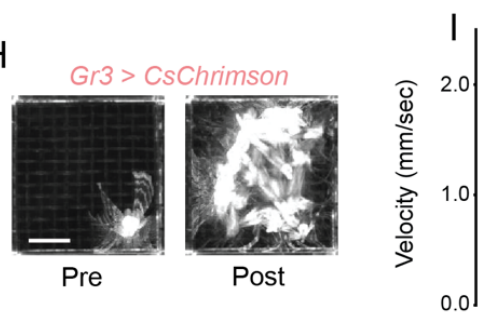
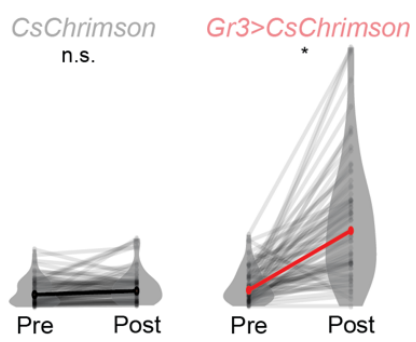

$\mathrm{K}$
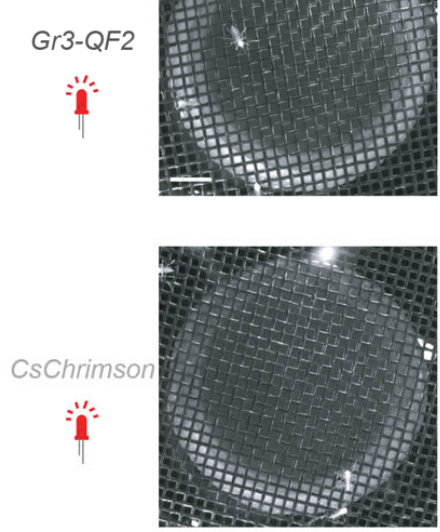

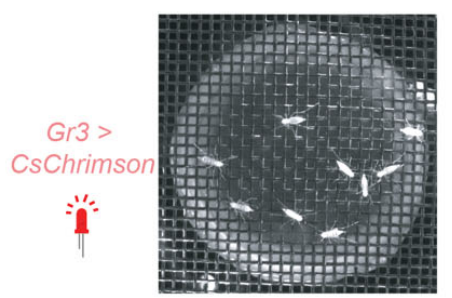

L
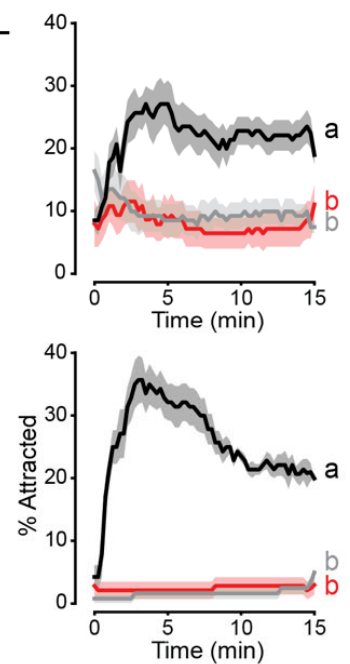

M
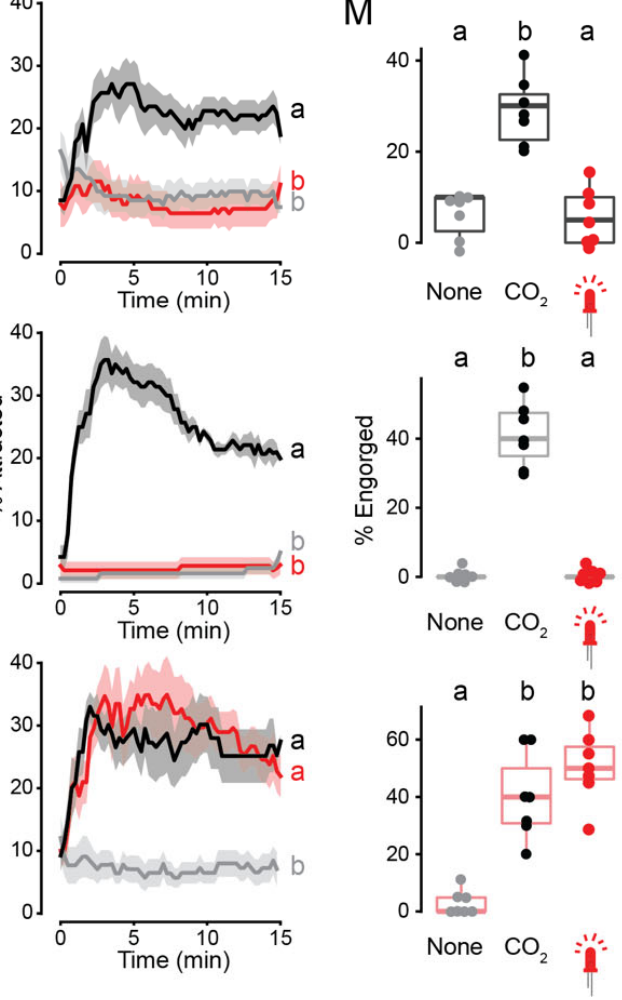

Figure 1. Optogenetic control of mosquito host seeking and blood feeding

(A) Schematic of human host cues experienced by a host-seeking mosquito over time. (B) Schematic of genetic reagents used for optogenetic activation of $\mathrm{CO}_{2}$-sensitive Gr3 sensory neurons. (C) Female Aedes aegypti, gray boxes indicating antenna (top) and maxillary palp (bottom). Photo: Alex Wild. (D) Intrinsic tdTomato fluorescence of whole mounted Gr3 > CsChrimson female mosquito antenna and maxillary palp. Scale bar: $50 \mu \mathrm{m}$. (E) Maximum-intensity projections of confocal Z-stacks of antennal lobes in the right-brain hemisphere of the indicated genotype with immunofluorescent labeling of tdTomato (red) and the synaptic marker BRP (grayscale). Scale bar: $10 \mu \mathrm{m}$. (F-G) Diagram (F) and stimulus protocol (G) of optogenetic behavior assay for mosquito movement. (H) Time maximum projection of a single mosquito in the assay in (F) for $30 \mathrm{sec}$ pre- (left) and post- (right) stimulus. Scale bar: $0.5 \mathrm{~cm}$. (I) Velocity of individual 
mosquitoes of the indicated genotypes $30 \mathrm{sec}$ pre- and post-stimulus onset. Data are plotted as mean of individual mosquitoes (thin gray lines) with median across individuals indicated with thick black or red line $\left({ }^{*} P<0.0001\right.$, Wilcoxon signed rank test with Holm's correction for multiple comparison, n.S., not significant, $n=70$ mosquitoes/genotype). (J) Schematic of opto-membrane feeder (top) and stimulus protocol (bottom). (K) Still images of mosquitoes of the indicated genotype underneath the warm blood meal approximately 7 min after the start of red-light stimulation. Scale bar: $1 \mathrm{~cm}$. (L) Occupancy of mosquitoes on warm blood meal in the opto-membrane feeder. Data are plotted as mean (line) \pm S.E.M. (shading). Data labeled with different letters are significantly different at the 5 min timepoint $(P<0.05$, Kruskal-Wallis test followed by Nemenyi post-hoc tests; $n=6-7$ trials per genotype/stimulus combination, 18-21 mosquitoes/trial). (M) Percent of mosquitoes visually scored as engorged at the conclusion of the experiment in L. Data are plotted as dot-box plots (median: horizontal line, interquartile range: box, 1.5 times interquartile range: whiskers. Data labeled with different letters are significantly different $(P<0.05$, Kruskal-Wallis test followed by Nemenyi post-hoc tests; $\mathrm{n}=7$ trials per genotype/stimulus combination and $18-21$ mosquitoes/trial).

The use of fictive $\mathrm{CO}_{2}$ delivered optogenetically was critical for studying the internal state of the mosquito after these transient host cues. Delivery and removal of real $\mathrm{CO}_{2}$ necessitates constant air flow, which is itself an important sensory cue for insects. Optogenetic activation of the $\mathrm{CO}_{2}$ sensory neurons allowed us to separate the effect of $\mathrm{CO}_{2}$ from this continuous stimulus as well as control it with greater precision. We delivered 5 -second red light pulses and heat increases to simulate brief $\mathrm{CO}_{2}$ and human body heat stimuli (Figure 2B). Mosquitoes responded to individual heat and fictive $\mathrm{CO}_{2}$ stimuli with elevated walking, flying, and probing (Figure 2E-H, Figure S1). The response to heat alone was dominated by probing and returned to baseline after about one minute $\left(\mathrm{t}_{1 / 2}\right.$ probing $\left.=0.4 \mathrm{~min}\right)$. In contrast, fictive $\mathrm{CO}_{2}$ alone caused an immediate flight and probing response followed by sustained walking, flying, and probing (Figure $2 \mathrm{H}$, Figure $\mathrm{S} 1$, Video S1). This sustained response took approximately 15 minutes to return to baseline $\left(\mathrm{t}_{1 / 2}\right.$ probing $\left.=3.9 \mathrm{~min}\right)$. The long duration of the response to $\mathrm{CO}_{2}$ is reminiscent of persistent internal states in other organisms (23-26).

These observations of mosquito behavior could reflect an internal state specific to host-seeking behavior or a general arousal state elicited by many sensory stimuli. Like $\mathrm{CO}_{2}$, bright light is also an arousal signal in mosquitoes (27) so we asked whether bright light induces a long-lasting behavior state. We used wild-type mosquitoes to test the response to this visual startle stimulus without the activation of

neurons expressing CsChrimson. A bright green light stimulus induced a brief response dominated by walking ( $\mathrm{t}_{1 / 2}$ walking $=0.4 \mathrm{~min}$ ), much shorter than the response to fictive $\mathrm{CO}_{2}$ (Figure 2l,K).

While $\mathrm{CO}_{2}$ and heat elicit the blood-feeding program required for females to produce eggs, mosquitoes possess a second, distinct feeding program for ingestion of flower nectar for energy $(28,29)$. We asked whether optogenetic stimulation of Gr4 sensory neurons that detect sugars in flower nectar evoked a sustained behavior response in mosquitoes as with optogenetic activation of the $\mathrm{CO}_{2}$ sensory neurons. We found that fictive sugar elicited a response composed largely of probing that was of shorter duration than that of fictive $\mathrm{CO}_{2}\left(\mathrm{t}_{1 / 2}\right.$ probing $\left.=1.5 \mathrm{~min}\right)($ Figure $2 \mathrm{~J}, \mathrm{~L})$.

The temporal resolution of our assays allowed us to understand precisely how mosquitoes integrate $\mathrm{CO}_{2}$ and heat to affect their behavior. We focused on the first 15 seconds after stimulus onset during which the greatest behavior responses are seen. We found that the heightened probing response seen when the stimuli were presented together was roughly additive with respect to the individual stimuli (Figure 2M, Figure S2). In contrast, flying was strongly suppressed. This demonstrates that multimodal integration of host cues biases action selection away from long-range flight and toward a short-range probing behavior.

Heat is a host cue but also may be experienced by the mosquito under other environmental contexts. We compared the integration of heat with green light and fictive sugar to see if they are integrated similarly to the host cue $\mathrm{CO}_{2}$. In contrast to the integration of host cues, probing was suppressed when non-host cues were presented together (Figure 2M, Figure S2). This demonstrates that the mosquito nervous system uses different computations for the integration of host cues and non-host cues.

\section{The $\mathrm{CO}_{2}$-evoked persistent state is specific to host} seeking

The fact that bright green light and fictive sugar stimuli elicited briefer responses suggested that the prolonged response to fictive $\mathrm{CO}_{2}$ is specific to host seeking. If true, it should be modulated in the same ways that host-seeking behavior is modulated. Host seeking in Aedes aegypti is suppressed after a female takes a blood meal, only returning after she lays eggs several days later $(30,31)$. We allowed female mosquitoes to blood feed on a human arm and then assayed their behavior four days later. We compared these animals to non-blood-fed females (Figure $3 A, B, D, E)$. We found that blood-fed females completely lost their response to fictive $\mathrm{CO}_{2}$ and nearly completely lost their response to heat. This demonstrates that in addition to losing the persistent state, blood-fed females lose even brief responses to host cues.

Unlike females, male mosquitoes do not seek out hosts to feed on blood. Males do demonstrate a flight response to $\mathrm{CO}_{2}$ and are reported to congregate in the vicinity of humans where they mate with female mosquitoes $(32,33)$. We observed that male mosquitoes had minimal responses to heat, but substantial flight and walking responses to fictive $\mathrm{CO}_{2}$ (Figure 3C-E). However, the response to fictive $\mathrm{CO}_{2}$ was brief, decaying rapidly back to baseline $\left(t_{1 / 2}\right.$ probing $=0.4$ min). Thus, male mosquitoes possess an initial response to fictive $\mathrm{CO}_{2}$ but lack a prolonged response to this stimulus. This observation raised the possibility that the persistencebut not the initial response-is specifically regulated in a sexually dimorphic manner. 
bioRxiv preprint doi: https://doi.org/10.1101/2021.10.06.463436; this version posted October 7, 2021. The copyright holder for this preprint (which was not certified by peer review) is the author/funder, who has granted bioRxiv a license to display the preprint in perpetuity. It is made available under aCC-BY 4.0 International license.

A

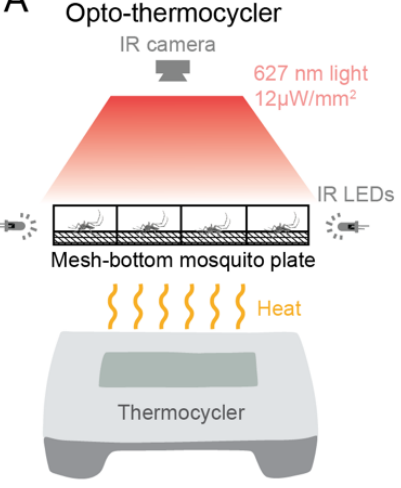

B
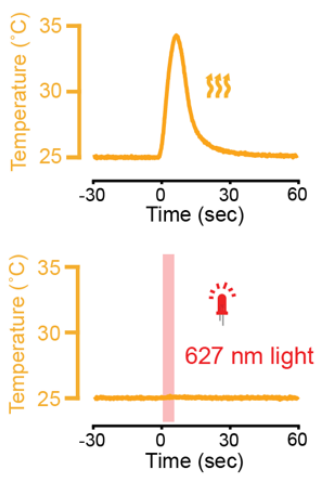

C

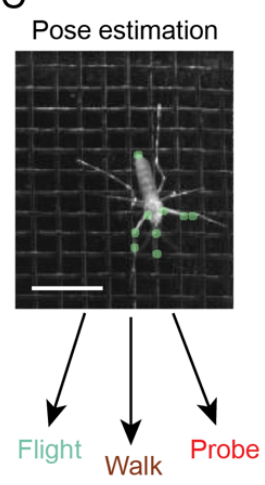

D Flight Walk Probe

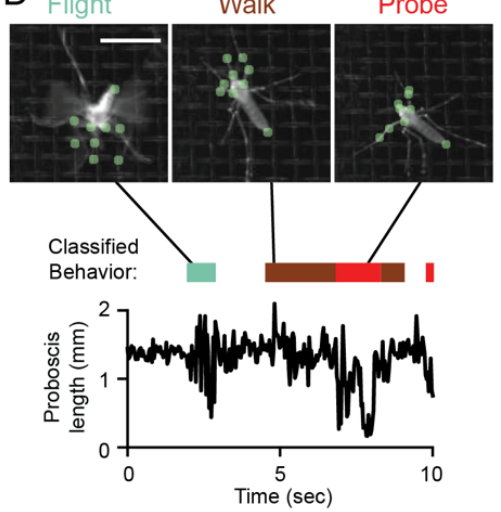

E Wild type Gr3 CsChrimson CsChrimsc

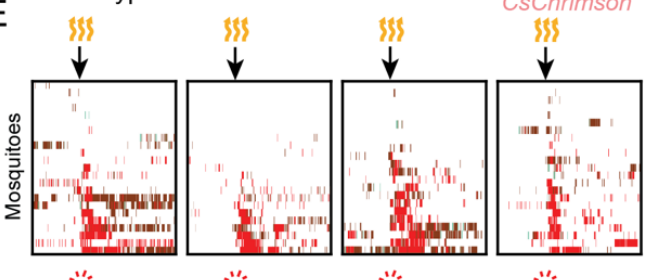

$\mathrm{F}$

G
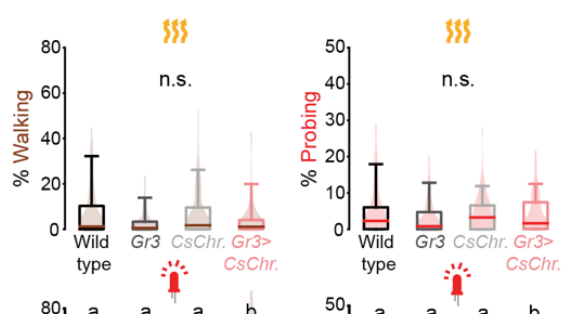

$\mathrm{H}$
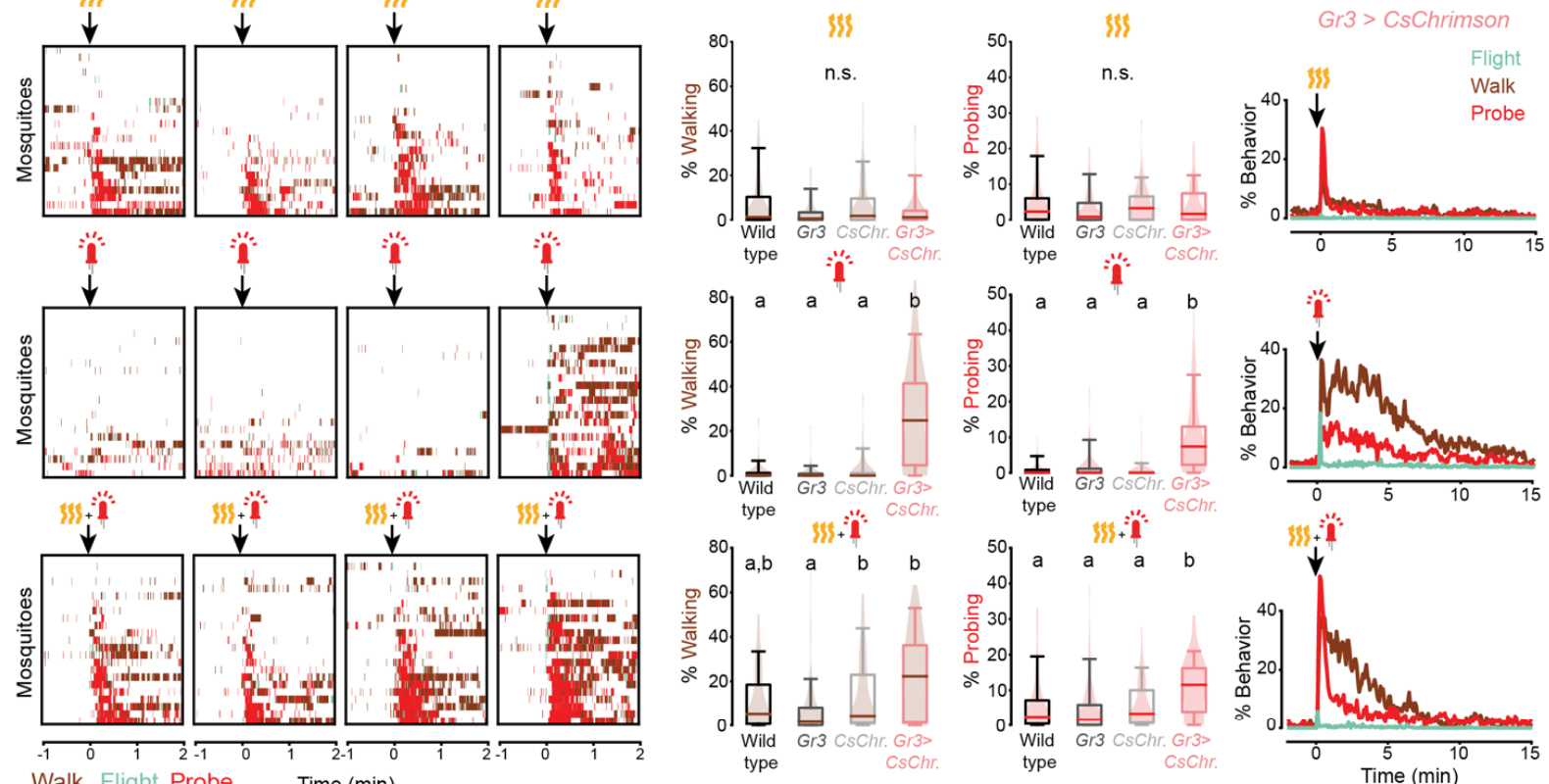

$$
\text { Walk Flight Probe Time (min) }
$$
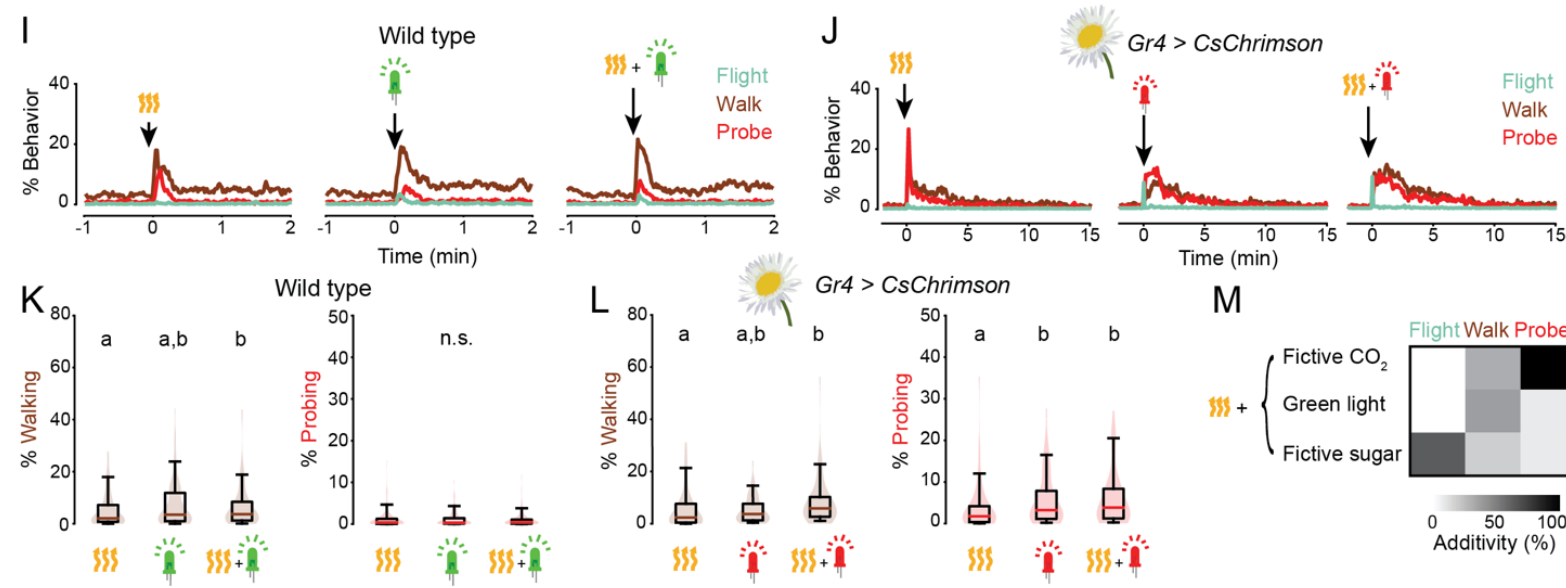

$\mathrm{M}$

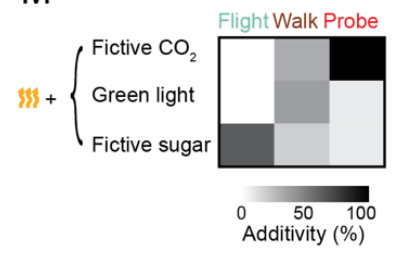

Figure 2. Fictive $\mathrm{CO}_{2}$ induces a persistent behavior state

(A-B) Schematic of opto-thermocycler assay (A) and stimuli delivered (B). (C) Still image of a mosquito with pose tracking of 9 points using Animal Part Tracker. Scale bar: $0.5 \mathrm{~cm}$. (D) Still images of a mosquito exhibiting the indicated classified behaviors (top). Representative plot of proboscis length with classified behavior superimposed (bottom). Scale bar: $0.5 \mathrm{~cm}$. (E) Ethograms of individual mosquitoes of the indicated genotypes. Data show $1 \mathrm{~min}$ before and an excerpt of the $2 \mathrm{~min}$ after the indicated stimuli from a 20 min experiment. Each row represents data from one mosquito. The experiment comprised a total of $n=68-70$ mosquitoes/condition. All data were sorted by probing, and every third mosquito $(n=22-23)$ was selected for display here for clarity. $(F-G)$ Quantification of walking $(F)$ and probing $(\mathrm{G})$ behavior exhibited by individual mosquitoes from the experiment in $(E)$ during the 5 min after stimulus onset. Data are plotted as violin-box plots (median: horizontal line, interquartile range: box, $5^{\text {th }}$ and $95^{\text {th }}$ percentiles indicated: whiskers). Data labeled with different 
letters are significantly different $(P<0.05$, Kruskal-Wallis test followed by Nemenyi post-hoc tests, n.s., not significant, $\mathrm{n}=68-70$ mosquitoes/genotype, 1 stimulus per trial). (H) Plot of percent individual Gr3 > CsChrimson mosquitoes exhibiting the indicated behavior from 2 min before to $15 \mathrm{~min}$ after stimulus onset. Data from experiment in (E). (I-J) Plot of percent individual wild type (I) and Gr4 > CsChrimson (J) mosquitoes exhibiting the indicated behavior from $1 \mathrm{~min}$ before to 2 min after stimulus onset (I) or 2 min before to 15 min after stimulus onset $(\mathrm{J})$ excerpted from a 20 min experiment. (I: $\mathrm{n}=140$ mosquitoes, average of 2 stimulus presentations/mosquito; J: $n=69$ mosquitoes, average of 3 stimulus presentations/mosquito). Flower image used for Gr4 > CsChrimson indicates plant nectar is source of sugar. (K, L) Quantification $(\mathrm{I})$ and $(\mathrm{J})$ for 5 min after stimulus onset. Data are plotted as violin-box plots (median: horizontal line, interquartile range: box, $5^{\text {th }}$ and $95^{\text {th }}$ percentiles: whiskers). Distribution represents individual mosquitoes, averaged over multiple stimulus presentations. Data labeled with different letters are significantly different $(P<0.05$, Friedman test followed by Nemenyi post-hoc tests, n.s., not significant). (M) Median additivity of heat and the indicated stimuli presented simultaneously. Additivity of $100 \%$ corresponds to the case when combined stimuli equal the sum of responses to individual stimuli. Data from (E-L). See also Figure S2.

We have previously shown that fruitless mutant male mosquitoes gain strong attraction to human odor (17). We asked whether these fruitless mutant males have an altered response to fictive $\mathrm{CO}_{2}$ stimuli. First, we confirmed that fruitless mutant males lacking Gr3 > CsChrimson did not respond to red light, as expected (Figure 3F). fruitless heterozygotes receiving fictive $\mathrm{CO}_{2}$ showed a brief response $\left(t_{1 / 2}\right.$ probing $=0.4 \mathrm{~min})($ Figure $3 \mathrm{G}, \mathrm{I}, \mathrm{J})$, similar to the response we saw for wild type males (Figure $3 \mathrm{C}$ ). By comparison, fruitless mutant males receiving fictive $\mathrm{CO}_{2}$ showed a strong and sustained response for minutes after the stimulus ( $t_{1 / 2}$ probing $=1.7 \mathrm{~min}$ ) (Figure $3 \mathrm{H}-\mathrm{J})$. Thus, fruitless regulates the persistent host-seeking state by suppressing this behavior in males. We note that the duration of the sustained response of fruitless mutant males is shorter than in females, suggesting additional sexually dimorphic factors may regulate this internal state.

Taken together, these results demonstrate that blood-fed females and males, which do not engage in blood-feeding behavior, lack sustained responses to brief pulses of fictive $\mathrm{CO}_{2}$. Males lacking the gene fruitless were previously reported to show strong attraction to human scent (17) and we show here that they also demonstrate strong and long-lasting responses to fictive $\mathrm{CO}_{2}$ reminiscent of females. This raises the possibility that the persistent state is the behavioral mechanism by which the goal of blood feeding is sustained in the female mosquito.

\section{Mosquitoes integrate sensory cues for minutes}

Motivation consists of two components: increased arousal and directed action toward a goal. We have demonstrated that fictive $\mathrm{CO}_{2}$ induces a prolonged increase in movement and the probing behavior that immediately precedes blood feeding. We asked whether the persistent state induced by a brief pulse of fictive $\mathrm{CO}_{2}$ can influence the response to body heat and ultimately if it can induce blood feeding many minutes afterwards.

First, we tested whether fictive $\mathrm{CO}_{2}$ primes subsequent responses to heat (Figure 4A). Because $\mathrm{CO}_{2}$ is highly volatile, mosquitoes likely sense this cue before body heat in naturalistic host-seeking settings. When we presented heat first followed by fictive $\mathrm{CO}_{2}$, relatively small behavioral responses were evoked (Figure 4B-C). However, when fictive $\mathrm{CO}_{2}$ was presented simultaneously with heat or 15 or 60 seconds prior to heat, larger walking and probing responses were seen (Figure 4B-C). This suggests that mosquitoes respond most strongly to the naturalistic temporal order of these host cues.

Next, we asked how long prior to the heat stimulus fictive $\mathrm{CO}_{2}$ can boost the response (Figure 4D). Compared to heat alone, walking and probing were increased when a fictive $\mathrm{CO}_{2}$ stimulus was presented up to four minutes prior (Figure $4 \mathrm{E}-\mathrm{F}$ ). This demonstrates that in addition to the sustained behavior response, fictive $\mathrm{CO}_{2}$ increases the response to heat for minutes afterward.

Once a mosquito pierces the skin of a human host, taste cues present in the blood guide the decision to engorge. Because this is the final goal of host-seeking behavior, we wondered whether mosquitoes in the fictive $\mathrm{CO}_{2}$-triggered persistent state had altered responses to both heat and taste stimuli. To test this, we designed an opto-feeder assay that incorporated a thin sheet of a blood meal mimic located between the thermocycler heating element and the mesh below the mosquito, allowing it to be rapidly heated and cooled (Figure 4G-H). We used a solution of ATP in saline, which has previously been shown to be a highly palatable meal that induces females to engorge in the same manner as blood $(29,34)$. This allowed us to test how the prolonged arousal state induced by fictive $\mathrm{CO}_{2}$ influences the decision of the mosquito to feed on a blood meal mimic.

First, we explored the temporal relationship of host stimuli to test whether the naturalistic order of $\mathrm{CO}_{2}$, heat, and taste stimuli elicits greater rates of blood feeding. When fictive $\mathrm{CO}_{2}$ was presented along with heating of the meal for 10 minutes, many females fed to repletion (Figure 4I). To test the temporal order of these cues, we offered fictive $\mathrm{CO}_{2}$ and a meal that was only warmed for several minutes, resulting in reduced feeding levels (Figure 4I). When the order was swapped and females were briefly offered the warm meal prior to fictive $\mathrm{CO}_{2}$, very few females fed (Figure 4I). This suggests that mosquitoes feed at the highest rates when they receive a $\mathrm{CO}_{2}$ stimulus prior to heat and taste stimuli. 
bioRxiv preprint doi: https://doi.org/10.1101/2021.10.06.463436; this version posted October 7, 2021. The copyright holder for this preprint (which was not certified by peer review) is the author/funder, who has granted bioRxiv a license to display the preprint in perpetuity. It is made available under aCC-BY 4.0 International license.

A

B
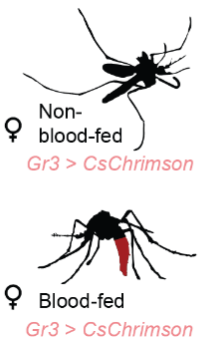

C

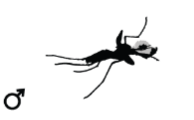

Gr3 > CsChrimson
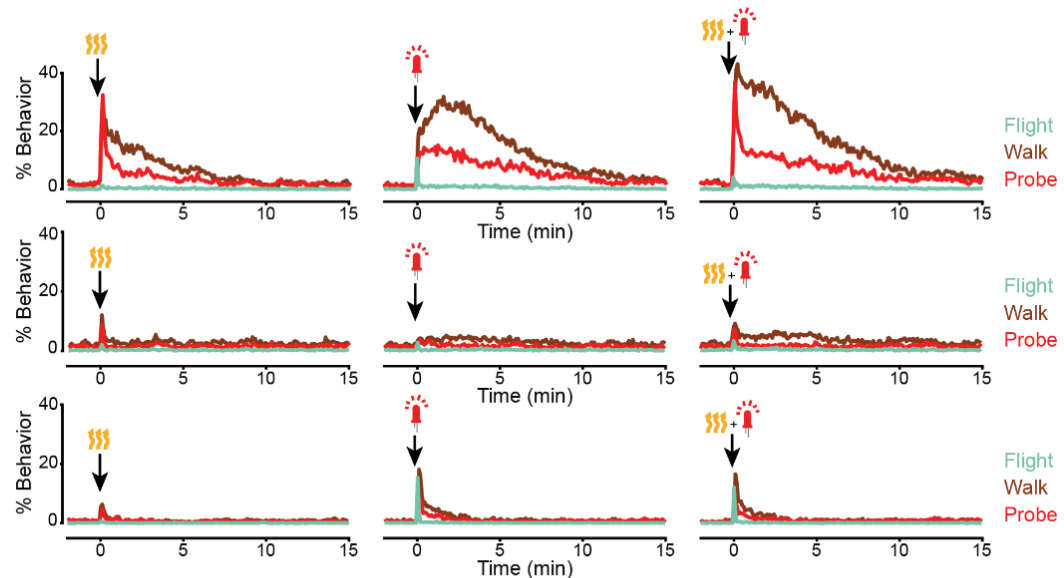

D

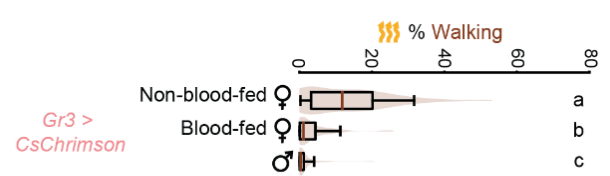

$\mathrm{E}$

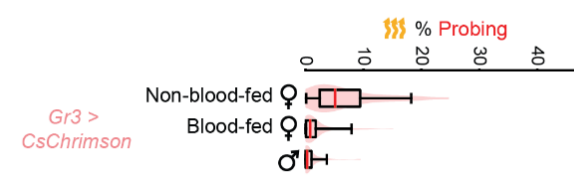

$\mathrm{F}$

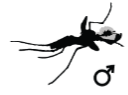

fruitless $^{\Delta M} /$ fruitless $^{\Delta M-\text { tdTomato }}$

G

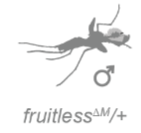

Gr3 > CsChrimson

$\mathrm{H}$
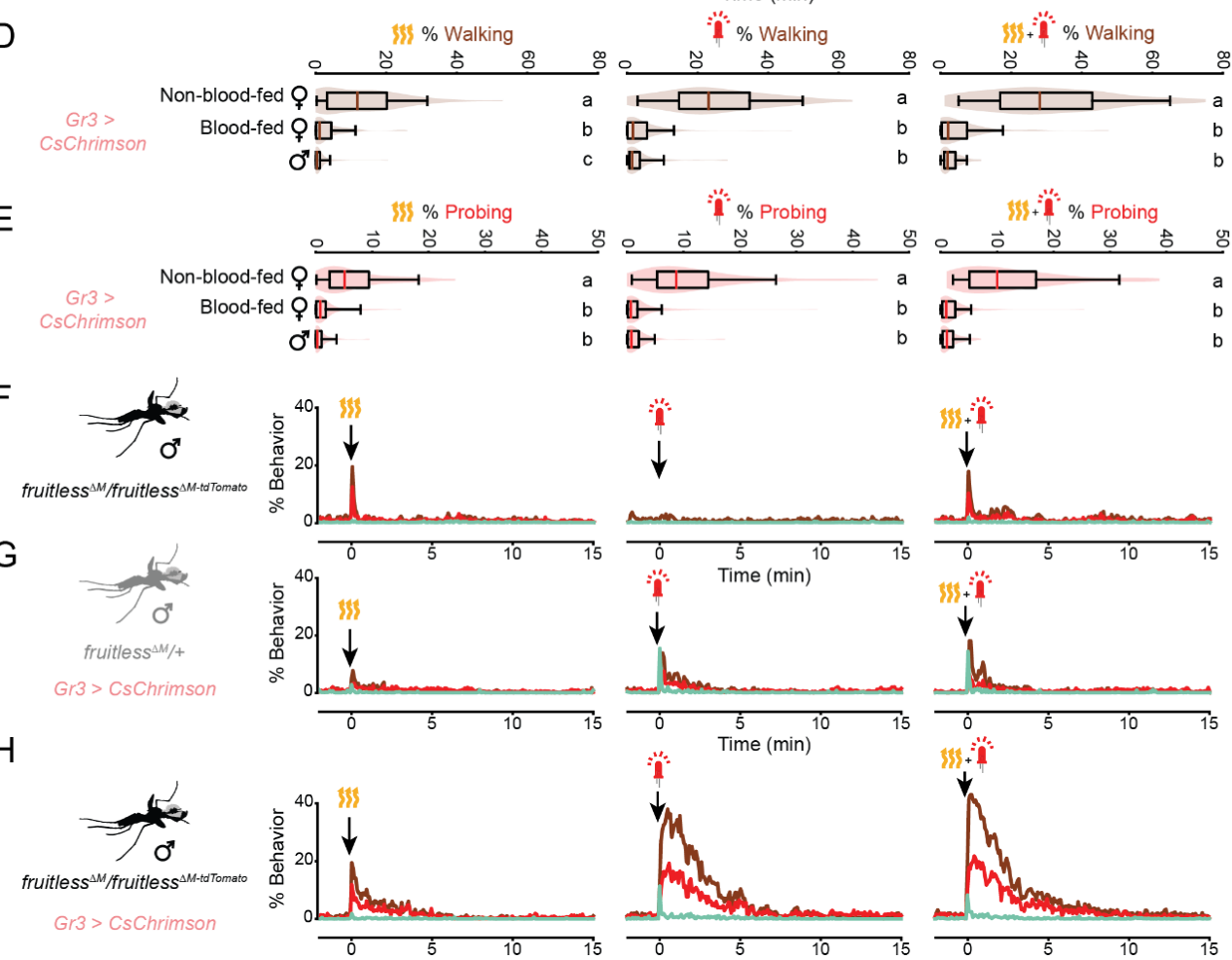

"i" \% Probing
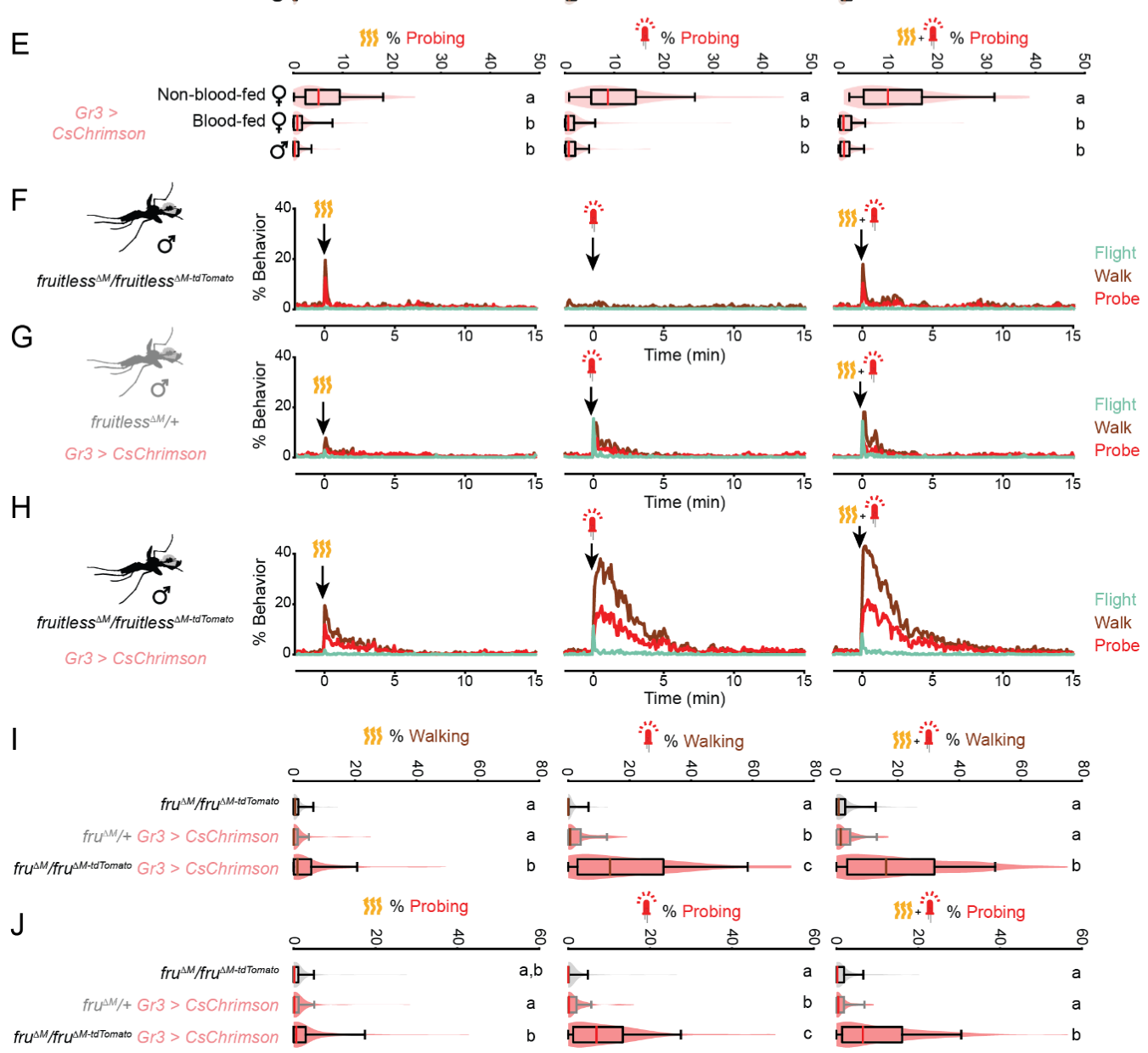

Figure 3. The persistent state is specific to host seeking

(A-C) Response of non-blood-fed female (A), blood-fed female (B), and male (C) Gr3 > CsChrimson mosquitoes to the indicated stimuli, plotting each behavior from $2 \mathrm{~min}$ before to $15 \mathrm{~min}$ after stimulus onset ( $\mathrm{n}=98 / \mathrm{group}$, average of 3 stimulus presentations/mosquito). (DE) Quantification of walking (D) and probing (E) from data in A-C for 5 min after stimulus onset. (F-H) The behavioral response of males of the indicated genotype for $15 \mathrm{~min}$ after the indicated stimuli, plotting each behavior from 2 min before to 15 min after stimulus onset ( $\mathrm{n}=97-98 /$ genotype, average of 3 stimulus presentations/mosquito). (I-J) Quantification of walking (I) and probing (J) from data in F-H for 5 min after stimulus onset. In (D-E) and (I-J), data are plotted as violin-box plots (median: horizontal line, interquartile range: box, 5th and 95th percentiles: whiskers). The distribution represents individual mosquitoes, averaged over multiple stimulus presentations. Data labeled with different letters are significantly different $(P<0.05$, Kruskal-Wallis test followed by Nemenyi post-hoc tests, n.s., not significant). 
bioRxiv preprint doi: https://doi.org/10.1101/2021.10.06.463436; this version posted October 7, 2021. The copyright holder for this preprint (which was not certified by peer review) is the author/funder, who has granted bioRxiv a license to display the preprint in perpetuity. It is made available under aCC-BY 4.0 International license.

A

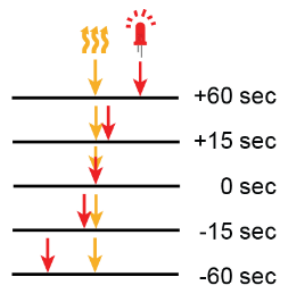

D

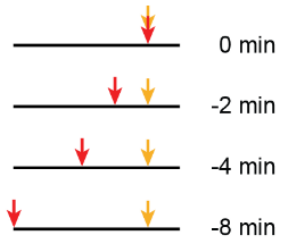

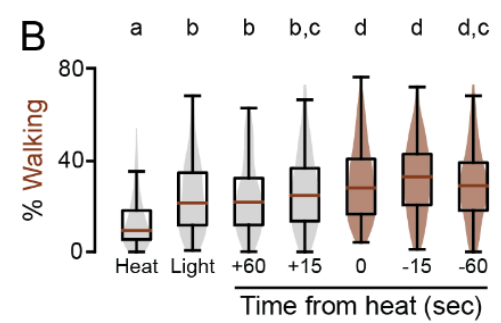

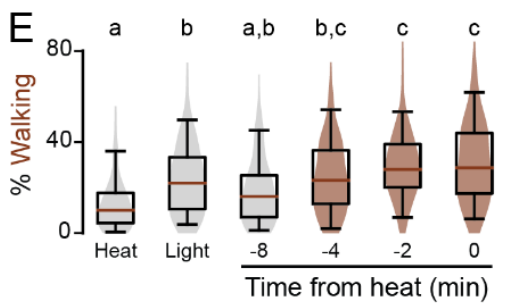

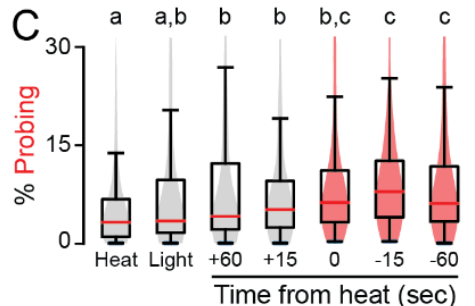

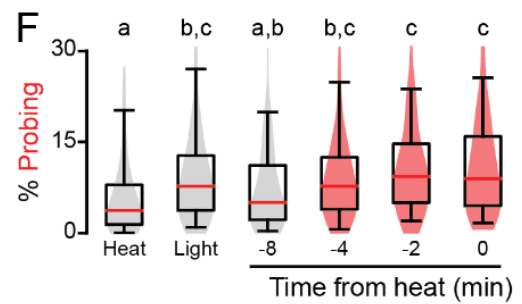

$\mathrm{J}$
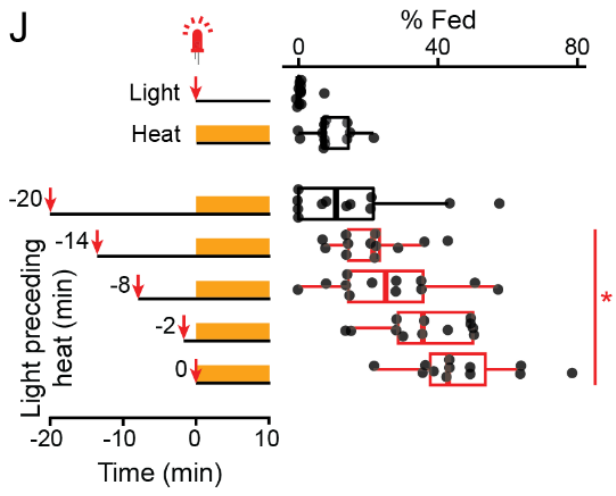
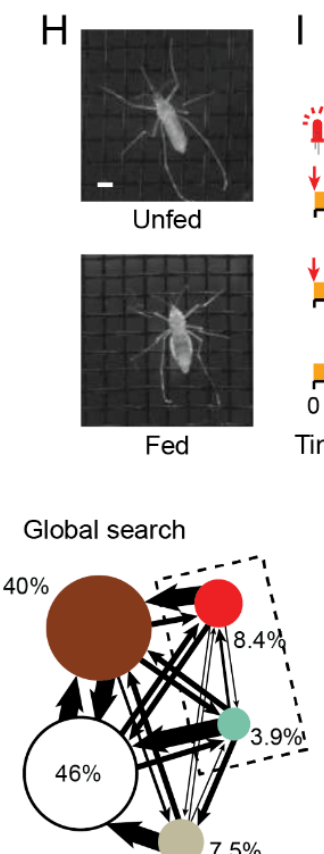

L

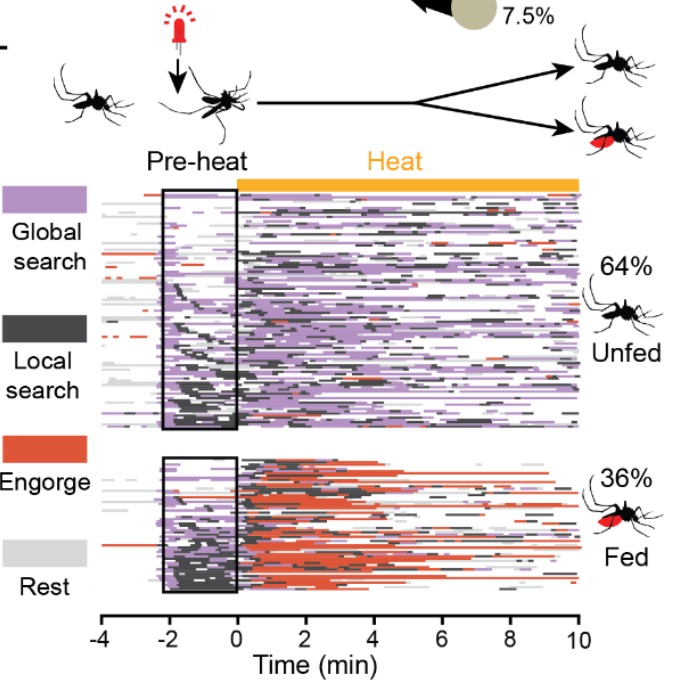

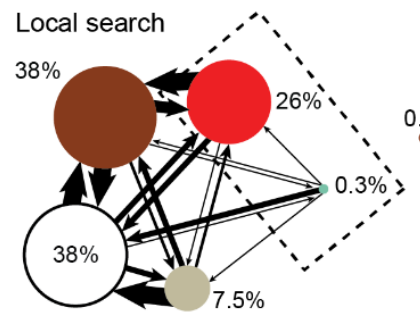

Engorge
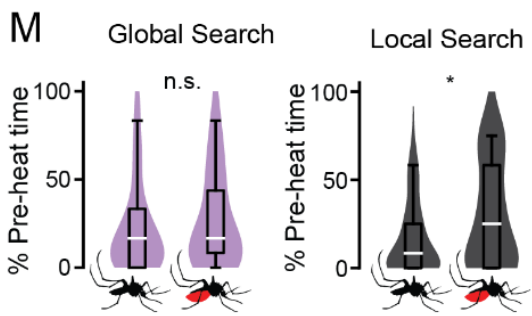

$\mathrm{N}$
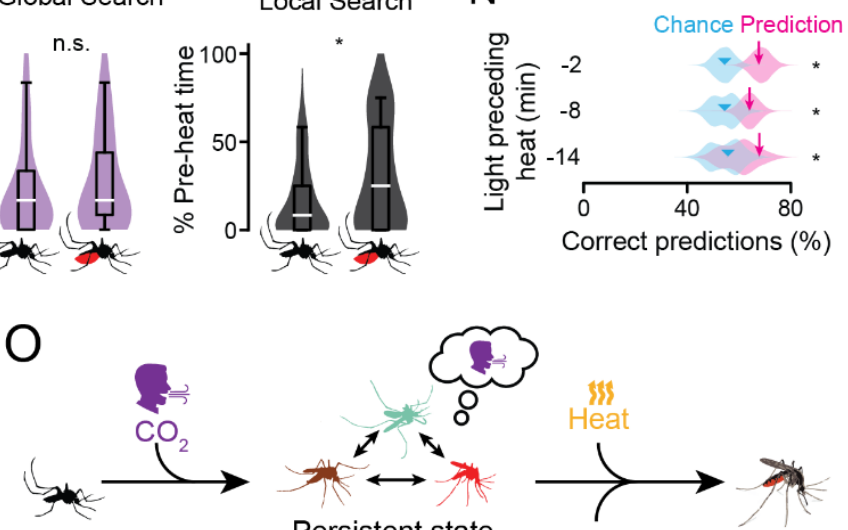

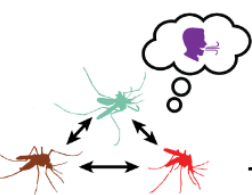

Persistent state $\leq 14$ minutes

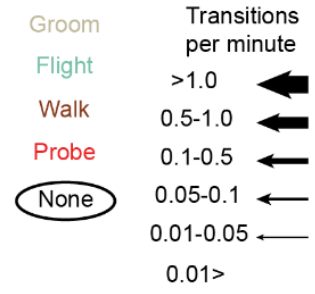

$0.01>$ 
$(P<0.05$, Friedman test followed by Nemenyi post-hoc tests). Data that are significantly different from heat or light are shaded in brown $(B, E)$ or red (C, F). (G) Schematic of opto-feeder assay. (H) Still images of unfed (top) and fed (bottom) mosquitoes. Scale bar: 1 mm. (I) Percent mosquitoes engorged in response to the indicated stimuli in the opto-feeder assay ( $\mathrm{n}=9$ trials/stimulus, $14 \mathrm{mosquitoes} /$ trial). Data labeled with different letters are significantly different $(P<0.05$, ANOVA followed by Tukey's post-hoc test). (J) Percent mosquitoes engorged in response to the indicated cues in the opto-feeder assay (14 mosquitoes/trial $n=11$ trials/stimulus). Data in (I) and (J) are displayed as dot-box plots (median: horizontal line, interquartile range: box, 1.5 times interquartile range: whiskers). Dot-box plots in $\mathrm{J}$ with a red border signify data where combined light and heat stimuli are greater than the sum of individual stimuli ( ${ }^{*} P<0.05$, Student's $t-$ test, after adjustment for multiple comparisons using Holm's method). (K) Transition ethograms for each of the four states indicating the proportion of each behavior and the rate of transitions between them, with dashed rectangle highlighting difference between global and local search. See also Figure S3 and Figure S4. (L) Inferred states of 168 individual mosquitoes from the 2 min pre-heat stimulus from experiment in $(J)$, separated into those that were visually scored as unfed (top, $n=108$ ) or fed (bottom, $n=60$ ) at the end of the experiment. Each row represents data from one mosquito and data are sorted according to the amount of local search during the pre-heat period. White indicates none of the four states were inferred (i.e. the mosquito was primarily motionless). (M) Quantification of the percent of time mosquitoes spent in the indicated state during the $2 \mathrm{~min}$ pre-heat period. Data are plotted as violin-box plots (median: horizontal line, interquartile range: box, 5th and 95th percentiles: whiskers $\left({ }^{*} P<0.01\right.$, Mann-Whitney U-test, n.s., not significant). (N) Performance of a classifier trained on the proportion of each behavior in pre-heat period and used to predict feeding (magenta arrow) along with 10,000 bootstrapped classifiers (magenta violin plot). Chance value (cyan arrowhead) indicates the median performance of model on 10,000 shuffles (cyan violin plot) of the feeding data in $(\mathrm{J})$. ( $\mathrm{n}=166-168$ mosquitoes/stimulus, $P=2 \mathrm{e}-4,0.0246,0.0123$ for 2,8 , and 14 min preheat periods, respectively, bootstrapped z-test). (O) Summary of the persistent internal state for host-seeking behavior. Color of the mosquito silhouettes indicates the behavior depicted using the colors in (K).

Next, we asked how long after a brief pulse of fictive $\mathrm{CO}_{2}$ females would retain the motivation to feed on the blood meal mimic. When offered the warm meal without fictive $\mathrm{CO}_{2}$, few females fed. We then stimulated the females with fictive $\mathrm{CO}_{2}$ and heated the blood meal mimic either immediately or after a delay of $2,8,14$, or 20 minutes after fictive $\mathrm{CO}_{2}$. We hypothesized that if the fictive $\mathrm{CO}_{2}$ induced a persistent state of host seeking, it might trigger engorging behavior many minutes later. Indeed, fictive $\mathrm{CO}_{2}$ was able to potentiate feeding when presented up to 14 minutes prior to heating of the blood meal mimic (Figure 4J). Taken together, our results demonstrate that the persistent host-seeking state increases host-seeking behaviors and alters the response to sensory cues for many minutes after a brief fictive $\mathrm{CO}_{2}$ stimulus. We speculate that this reflects the amount of time a mosquito will pursue a host in a naturalistic setting before halting the search if it appears that the host is no longer nearby.

We noticed that there is considerable individual variation in how mosquitoes respond to the warm blood meal mimic after being activated by fictive $\mathrm{CO}_{2}$ (Figure 4J). We asked whether the behavior state of individual mosquitoes could be inferred over longer periods of time. We extracted 38 behavior parameters from the experiment in Figure $4 \mathrm{~J}$ from 30 second long time windows and used t-distributed stochastic neighbor embedding (tSNE) to visualize the relationships. This embedding revealed that mosquito behavior fell into four major states that we termed rest, global search, local search, and engorge (Figure 4K, Figure S3). These states were observed even when the length of the time window was varied over a 6-fold range (Figure S4). The states differed in the proportions of behaviors observed, the transition rates between them, and the total distance the mosquito traveled while in each state. We divided the data into those females that eventually fed or did not feed at the end of the experiment (Figure 4L). As expected, the engorge state was greatly enriched in mosquitoes that were categorized as fed, demonstrating that this method is effective at identifying longer-timescale behavior states (Figure 4L).
We asked whether the behavior state of the mosquito after fictive $\mathrm{CO}_{2}$ was associated with the future decision to feed on the warm blood meal mimic. We found that fed and unfed mosquitoes showed similar amounts of global search preheat but greater levels of local search for those that would later feed (Figure 4M). The local search state differs primarily by showing more probing and less flight than the global search state. To avoid potential distortions created by the 2dimensional embedding (35), we trained a linear classifier using only the four behaviors (groom, walk, flight, and probe). A classifier trained on the proportion of time mosquitoes spent in each behavior for 2 minutes after the light stimulus could predict which mosquitoes would later feed with above-chance accuracy (Figure $4 \mathrm{~N}$ ). Thus, the behavior state that individual mosquitoes enter in response to fictive $\mathrm{CO}_{2}$ reflects the likelihood of response to future sensory cues.

\section{DISCUSSION}

We investigated how female mosquitoes pursue humans by combining multisensory stimuli in time to achieve the goal of feeding on human blood. To precisely control the delivery of $\mathrm{CO}_{2}$, we generated optogenetic tools to deliver fictive $\mathrm{CO}_{2}$ and combined this with high-resolution behavior assays and machine learning analysis approaches. These experiments demonstrated that optogenetic activation of $\mathrm{CO}_{2}$ sensory neurons induced a long-lasting behavior state change (Figure 40). During this time mosquitoes had heightened responses to heat and were more likely to feed on a blood meal mimic even if encountered minutes after the $\mathrm{CO}_{2}$ stimulus.

Integration of sensory information over time allows the nervous system to optimize decision making for a particular goal (36). Testing whether predators use this approach during hunting requires a precise understanding of what the predator is sensing and when, precluding field studies. The challenge of delivering precise olfactory stimuli in turbulent air plumes means that most studies deliver volatile cues over long time periods. Nevertheless, some studies have found 
behavior responses altered for as long as a few seconds after encountering $\mathrm{CO}_{2}$ in mosquitoes and other olfactory stimuli in Drosophila $(13,37-39)$. Although this timescale of sensory integration is sufficient for upwind tracking, modeling suggests that integration over long timescales can maximize the information about the location of an olfactory stimulus (40). Here, we show that mosquitoes integrate olfactory, heat, and taste stimuli for at least 14 minutes, much longer than previously assumed.

Integration of human host cues from multiple sensory modalities is a hallmark of mosquito behavior, as well as a general strategy of other predators in pursuit of prey. Our techniques revealed a high-resolution understanding of how heat and $\mathrm{CO}_{2}$ were behaviorally integrated. The computations used to integrate these cues suppress flight and walking but promote probing and the subsequent decision to engorge. Thus, the mosquito uses multiple cues-as well as their timing - as stronger evidence of host presence than a single cue and biases its actions toward biting. The increased movement and the bias of actions toward a particular goal constitute an internal motivational state sustained over minutes-one specific to feeding on humans. Importantly, our results show that this internal state does not require constant flight behavior or a constant air flow stimulus, demonstrating that it is maintained by an internal mechanism rather than continuous sensory or reafferent stimuli.

In addition to examining population responses to host cues, we inferred the states of individual mosquitoes and found that they fell into four categories: rest, global search, local search, and engorge. Analogous local and global search states during food finding are found in other organisms from diverse clades $(23,41-44)$. After an animal senses taste or olfactory cues indicative of food, it performs localized movements characterized by increasing turn frequency to cover a restricted area. The broad phylogenetic distribution of this behavior raises the question of whether it is deeply conserved from a common ancestor or whether it represents convergent solutions to similar biological problems.

In principle, the long-duration characteristic of the hostseeking state could be generated by neurons at any point in the circuit. However, it is extensively documented in Aedes aegypti that $\mathrm{CO}_{2}$ sensory neurons show an accurate readout of $\mathrm{CO}_{2}$ levels over a large concentration range $(21,45)$. These neurons do not show adaptation or prolonged activity (21) and have similar responses in males and females (45). While we suspect based on precedents for persistent states observed in other systems that the persistent state is controlled in the central brain, we cannot exclude a contribution from the periphery. In mice, circuits originating in the central amygdala promote pursuit and attack during cricket hunting (46), but these behaviors appear time-locked to optogenetic activation and a circuit controlling hunting persistence has not been identified. We hypothesize that, as in mice, the mosquito host-seeking circuit contains a dissociable organization of motor programs for different stages of search, probing, and engorgement behaviors. These motor programs would presumably receive input from a central circuit controlling the persistent host-seeking state.

Mosquito host seeking shares some characteristics with other social and feeding states (23-26). It is long lasting, alters sensory responses in multiple modalities, and organizes actions to accomplish a goal that outlasts individual stimuli. Because persistent states occur over longer durations, they have the capacity to serve as a memory of past sensory information and direct behavior tasks toward completion. The pursuit of females by males during courtship behavior in Drosophila shows especially striking similarities. In response to female sensory cues or stimulation of a subset of fruitless neurons, male flies enter a state of increased courtship behaviors and lower thresholds for sensory cues from females $(26,47-49)$. This work suggests that circuits regulated by fruitless confer persistent behavioral states across different systems-male mating and female blood-feedingover hundreds of millions of years of insect evolution.

Mosquitoes are thought to have evolved the ability to feed on blood through a single gain at the base of their lineage (50). Their non-biting ancestors may have fed primarily on plants or other insects using piercing mouthparts (51). Therefore, the major innovation for blood feeding could have been host-seeking behavior with mosquito morphology "preadapted" for piercing skin. Our results suggest an interesting hypothesis regarding the evolution of host seeking. In Drosophila and other insects, fruitless-expressing neural circuits control male courtship (52-54), which also displays the properties of persistence and sexual dimorphism. These similarities suggest that mosquito evolution may have co-opted these properties of ancestral fruitless circuits to drive a novel feeding behavior.

This study illuminates why mosquitoes are such effective predators: they maintain the goal of blood feeding for minutes even in the absence of any additional positive stimuli or reinforcement. Because this state greatly outlasts individual sensory stimuli and integrates multiple modalities, any intervention that disrupts this internal drive state should be more effective than vector control measures that mask or disrupt any individual aspect of host-seeking.

\section{SUPPLEMENTAL MATERIALS}

Figures S1-S4 and Video S1 accompany the paper. Data File S1 includes information on the accuracy of the behavioral classifier and all raw data and statistical analysis in the paper.

\section{ACKNOWLEDGMENTS}

We thank Hessam Akhlaghpour, Josie Clowney, Emily Dennis, Ann Kennedy, Philip Kidd, and members of the Vosshall lab for comments on the manuscript. We thank Allen Lee, Alice Robie, and Kristin Branson for sharing their unpublished Animal Part Tracker and providing technical help with running it. We thank Jason Banfelder and Rebecca Bennett with software setup on the Rockefeller High Performance Computing Cluster; Jim Petrillo, Dan Gross, and Peer Strogies at the Rockefeller Precision Instrumentation Technologies facility for assistance with design and fabrication of behavior assays; Gloria Gordon and Libby Mejia for expert mosquito rearing; Tom Hindmarsh Sten, Veronica Jové, Gaby Maimon, Ben Matthews Chris Potter, Vanessa Ruta, and Nilay 
Yapici for discussions; Annie Handler, Jazz Weisman, and Ari Zolin for technical advice with optogenetics experiments; Ben Matthews, Meg Younger, and the Aedes Toolkit Group for access to unpublished strains; Cong Li for assisting with an early version of the individual state analysis; and Rob Harrell at the Insect Transformation Facility for embryo injections. Pilot experiments for this study used an optogenetic setup in the lab of Vanessa Ruta.

\section{FUNDING}

This work was funded by a Jane Coffin Childs postdoctoral Fellowship (T.R.S.), and a Kavli Neural Systems Institute Pilot Grant and Postdoctoral Fellowship (T.R.S.). L.B.V. is an investigator of the Howard Hughes Medical Institute.

\section{AUTHOR CONTRIBUTIONS}

T.R.S. performed all experiments and analyses in the paper with the exception of Figure 4I, which was performed by A.R.-V. The optogenetic mosquito lines were created and tested by T.R.S, and A.P., who also developed the rearing and experimental protocol. T.R.S and L.B.V. conceived the study, created the figures, and wrote the manuscript with input from all authors.

\section{DECLARATION OF INTERESTS}

The authors declare no competing interests.

\section{REFERENCES}

B. R. Anholt, D. Ludwig, J. B. Rasmussen, Optimal pursuit times: How long should predators pursue their prey? Theor Popul Biol 31, 453-464 (1987).

2. T. M. Williams, L. Wolfe, T. Davis, T. Kendall, B. Richter, Y. Wang, C. Bryce, G. H. Elkaim, C. C. Wilmers, Mammalian energetics. Instantaneous energetics of puma kills reveal advantage of felid sneak attacks. Science 346, 81-85 (2014).

3. B. Leal, E. Zamora, A. Fuentes, D. B. Thomas, R. K Dearth, Questing by tick larvae (Acari: Ixodidae): A review of the influences that affect off-host survival. Ann Entomol Soc Am 113, 425-438 (2020).

4. J. F. Bergan, P. Ro, D. Ro, E. I. Knudsen, Hunting increases adaptive auditory map plasticity in adult barn owls. J Neurosci 25, 9816-9820 (2005).

5. M. Mischiati, H. T. Lin, P. Herold, E. Imler, R. Olberg, A. Leonardo, Internal models direct dragonfly interception steering. Nature 517, 333-338 (2015).

6. $\quad$ E. S. Hobson, Feeding behavior in three species of sharks. Pac Sci 17, 171-194 (1963).

7. J. M. Gardiner, J. Atema, R. E. Hueter, P. J. Motta, Multisensory integration and behavioral plasticity in sharks from different ecological niches. PLoS One $\mathbf{9}$, e93036 (2014).

8. K. D. Lafferty, A. M. Kuris, Trophic strategies, animal diversity and body size. Trends Ecol Evol 17, 507-513 (2002).

9. A. E. Eiras, P. C. Jepson, Host location by Aedes aegypti (Diptera: Culicidae): a wind tunnel study of chemical cues. B Entomol Res 81, 151-160 (1991).

10. C. J. McMeniman, R. A. Corfas, B. J. Matthews, S. A. Ritchie, L. B. Vosshall, Multimodal integration of carbon dioxide and other sensory cues drives mosquito attraction to humans. Cell 156, 1060-1071 (2014).

11. M. Geier, O. J. Bosch, J. Boeckh, Influence of odour plume structure on upwind flight of mosquitoes towards hosts. J Exp Biol 202, 1639-1648 (1999).
12. T. Dekker, M. Geier, R. T. Carde, Carbon dioxide instantly sensitizes female yellow fever mosquitoes to human skin odours. J Exp Biol 208, 2963-2972 (2005).

13. T. Dekker, R. T. Carde, Moment-to-moment flight manoeuvres of the female yellow fever mosquito (Aedes aegypti L.) in response to plumes of carbon dioxide and human skin odour. J Exp Biol 214, 3480-3494 (2011).

14. F. van Breugel, J. Riffell, A. Fairhall, M. H. Dickinson, Mosquitoes use vision to associate odor plumes with thermal targets. Curr Biol 25, 2123-2129 (2015).

15. R. A. Corfas, L. B. Vosshall, The cation channel TRPA1 tunes mosquito thermotaxis to host temperatures. Elife 4, 10.7554/eLife.11750 (2015).

16. M. A. Koehl, The fluid mechanics of arthropod sniffing in turbulent odor plumes. Chem Senses 31, 93-105 (2006).

17. N. S. Basrur, M. E. De Obaldia, T. Morita, M. Herre, R. K. von Heynitz, Y. N. Tsitohay, L. B. Vosshall, Fruitless mutant male mosquitoes gain attraction to human odor. Elife 9, (2020).

18. N. C. Klapoetke, Y. Murata, S. S. Kim, S. R. Pulver, A. Birdsey-Benson, Y. K. Cho, T. K. Morimoto, A. S. Chuong, E. J. Carpenter, Z. Tian, J. Wang, Y. Xie, Z. Yan, Y. Zhang, B. Y. Chow, B. Surek, M. Melkonian, V. Jayaraman, M. Constantine-Paton, G. K. Wong, E. S. Boyden, Independent optical excitation of distinct neural populations. Nat Methods 11, 338-346 (2014).

$19 . \quad$ C. J. Potter, B. Tasic, E. V. Russler, L. Liang, L. Luo, The $Q$ system: a repressible binary system for transgene expression, lineage tracing, and mosaic analysis. Cell 141, 536-548 (2010).

20. M. A. Younger, M. Herre, A. R. Ehrlich, Z. Y. Gong, Z. N. Gilbert, S. Rahiel, B. J. Matthews, L. B. Vosshall, Noncanonical odor coding ensures unbreakable mosquito attraction to humans. bioRxiv, DOI

2020.2011.2007.368720 (2020).

21. A. J. Grant, B. E. Wigton, J. G. Aghajanian, R. J. O'Connell, Electrophysiological responses of receptor neurons in mosquito maxillary palp sensilla to carbon dioxide. J Comp Physiol [A] 177, 389-396 (1995).

22. M. Kabra, A. A. Robie, M. Rivera-Alba, S. Branson, K. Branson, JAABA: interactive machine learning for automatic annotation of animal behavior. Nat Methods 10, 64-67 (2013).

23. J. C. Marques, M. Li, D. Schaak, D. N. Robson, J. M. Li, Internal state dynamics shape brainwide activity and foraging behaviour. Nature 577, 239-243 (2020).

24. S. W. Flavell, N. Pokala, E. Z. Macosko, D. R. Albrecht, J. Larsch, C. I. Bargmann, Serotonin and the neuropeptide PDF initiate and extend opposing behavioral states in C. elegans. Cell 154, 1023-1035 (2013).

25. K. Asahina, K. Watanabe, B. J. Duistermars, E. Hoopfer, C. R. Gonzalez, E. A. Eyjolfsdottir, P. Perona, D. J. Anderson, Tachykinin-expressing neurons control malespecific aggressive arousal in Drosophila. Cell 156, 221235 (2014).

26. T. Hindmarsh Sten, R. Li, A. Otopalik, V. Ruta, Sexual arousal gates visual processing during Drosophila courtship. Nature 595, 549-553 (2021).

$27 . \quad$ L. O. Araripe, J. R. A. Bezerra, G. Rivas, R. V. Bruno, Locomotor activity in males of Aedes aegypti can shift in response to females' presence. Parasit Vectors 11, 254 (2018).

28. C. Lahondere, C. Vinauger, R. P. Okubo, G. H. Wolff, J. K. Chan, O. S. Akbari, J. A. Riffell, The olfactory basis of orchid pollination by mosquitoes. Proc Natl Acad Sci U S A 117, 708-716 (2020). 
29. V. Jové, Z. Gong, F. J. H. Hol, Z. Zhao, T. R. Sorrells, T. S. Carroll, M. Prakash, C. S. McBride, L. B. Vosshall, Sensory discrimination of blood and floral nectar by Aedes aegypti mosquitoes. Neuron 108, 1163-1180 (2020).

30. M. Klowden, Initiation and termination of host-seeking inhibition in Aedes aegypti during oöcyte maturation. $J$ Insect Physiol 27, 799-803 (1981).

31. L. B. Duvall, N. S. Basrur, H. Molina, C. J. McMeniman, L. B. Vosshall, A peptide signaling system that rapidly enforces paternity in the Aedes aegypti mosquito. Curr Biol 27, 3734-3742 (2017).

32. B. J. Matthews, C. S. McBride, M. DeGennaro, O. Despo, L. B. Vosshall, The neurotranscriptome of the Aedes aegypti mosquito. BMC Genomics 17, 32 (2016).

33. W. K. Hartberg, Observations on the mating behaviour of Aedes aegypti in nature. Bull World Health Organ 45, 847-850 (1971).

34. R. Galun, Y. Avi-Dor, M. Bar-Zeev, Feeding response in Aedes aegypti: Stimulation by adenosine triphosphate. Science 142, 1674-1675 (1963).

35. T. Chari, J. Banerjee, L. Pachter, The specious art of single-cell genomics. bioRxiv, DOI 10.1101/2021.1108.1125.457696 (2021).

36. K. Körding, Decision theory: what "should" the nervous system do? Science 318, 606-610 (2007).

37. E. Alvarez-Salvado, A. M. Licata, E. G. Connor, M. K. McHugh, B. M. King, N. Stavropoulos, J. D. Victor, J. P. Crimaldi, K. I. Nagel, Elementary sensory-motor transformations underlying olfactory navigation in walking fruit-flies. Elife 7, (2018).

38. M. Demir, N. Kadakia, H. D. Anderson, D. A. Clark, T. Emonet, Walking Drosophila navigate complex plumes using stochastic decisions biased by the timing of odor encounters. Elife 9, (2020).

39. R. Pang, F. van Breugel, M. Dickinson, J. A. Riffell, A. Fairhall, History dependence in insect flight decisions during odor tracking. PLoS Comput Biol 14, e1005969 (2018).

40. M. Vergassola, E. Villermaux, B. I. Shraiman, 'Infotaxis' as a strategy for searching without gradients. Nature 445, 406-409 (2007).

41. W. M. Hamner, S. W. Strand, G. I. Matsumoto, P. P. Hamner, Ethological observations on foraging behavior of the ctenophore Leucothea sp. in the open sea. Limnol Oceanogr 32, 645-652 (1987).

42. D. Kuefler, T. Avgar, J. M. Fryxell, A. Knapp, Densityand resource-dependent movement characteristics in a rotifer. Functional Ecology 27, 323-328 (2013).

43. A. Lopez-Cruz, A. Sordillo, N. Pokala, Q. Liu, P. T. McGrath, C. I. Bargmann, Parallel multimodal circuits control an innate foraging behavior. Neuron 102, 407419 e408 (2019).

44. R. A. Corfas, T. Sharma, M. H. Dickinson, Diverse foodsensing neurons trigger idiothetic local search in Drosophila. Curr Biol 29, 1660-1668 e1664 (2019).

45. A. J. Grant, R. J. O'Connell, Age-related changes in female mosquito carbon dioxide detection. J Med Entomol 44, 617-623 (2007).

46. W. Han, L. A. Tellez, M. J. Rangel, Jr., S. C. Motta, X. Zhang, I. O. Perez, N. S. Canteras, S. J. ShammahLagnado, A. N. van den Pol, I. E. de Araujo, Integrated control of predatory hunting by the central nucleus of the amygdala. Cell 168, 311-324 e318 (2017).

47. H. K. Inagaki, Y. Jung, E. D. Hoopfer, A. M. Wong, N. Mishra, J. Y. Lin, R. Y. Tsien, D. J. Anderson, Optogenetic control of Drosophila using a red-shifted channelrhodopsin reveals experience-dependent influences on courtship. Nat Methods 11, 325-332 (2014).

48. E. J. Clowney, S. Iguchi, J. J. Bussell, E. Scheer, V. Ruta, Multimodal chemosensory circuits controlling male courtship in Drosophila. Neuron 87, 1036-1049 (2015).

49. Y. Jung, A. Kennedy, H. Chiu, F. Mohammad, A. Claridge-Chang, D. J. Anderson, Neurons that function within an integrator to promote a persistent behavioral state in Drosophila. Neuron 105, 322-333 e325 (2020).

50. B. M. Wiegmann, M. D. Trautwein, I. S. Winkler, N. B. Barr, J. W. Kim, C. Lambkin, M. A. Bertone, B. K. Cassel, K. M. Bayless, A. M. Heimberg, B. M. Wheeler, K. J. Peterson, T. Pape, B. J. Sinclair, J. H. Skevington, V. Blagoderov, J. Caravas, S. N. Kutty, U. Schmidt-Ott, G. E. Kampmeier, F. C. Thompson, D. A. Grimaldi, A. T. Beckenbach, G. W. Courtney, M. Friedrich, R. Meier, D. $\mathrm{K}$. Yeates, Episodic radiations in the fly tree of life. Proc Natl Acad Sci U S A 108, 5690-5695 (2011).

51. M. J. Lehane, The biology of blood-sucking in insects (chapter 2). (Cambridge University Press, Cambridge, 2005).

52. D. S. Manoli, M. Foss, A. Villella, B. J. Taylor, J. C. Hall, B. S. Baker, Male-specific fruitless specifies the neural substrates of Drosophila courtship behaviour. Nature 436, 395-400 (2005).

53. E. Demir, B. J. Dickson, fruitless splicing specifies male courtship behavior in Drosophila. Cell 121, 785-794 (2005).

54. R. C. Bertossa, L. van de Zande, L. W. Beukeboom, The fruitless gene in Nasonia displays complex sex-specific splicing and contains new zinc finger domains. Mol Biol Evol 26, 1557-1569 (2009).

55. M. DeGennaro, C. S. McBride, L. Seeholzer, T. Nakagawa, E. J. Dennis, C. Goldman, N. Jasinskiene, A. A. James, L. B. Vosshall, orco mutant mosquitoes lose strong preference for humans and are not repelled by volatile DEET. Nature 498, 487-491 (2013).

56. O. Riabinina, D. Task, E. Marr, C. C. Lin, R. Alford, D. A. O'Brochta, C. J. Potter, Organization of olfactory centres in the malaria mosquito Anopheles gambiae. Nat Commun 7, 13010 (2016).

57. D. L. Stern, Tagmentation-based mapping (TagMap) of mobile DNA genomic insertion sites. bioRxiv, DOI: 10.1101/037762 (2017).

58. B. J. Matthews, O. Dudchenko, S. B. Kingan, S. Koren, I. Antoshechkin, J. E. Crawford, W. J. Glassford, M. Herre, S. N. Redmond, N. H. Rose, G. D. Weedall, Y. Wu, S. S. Batra, C. A. Brito-Sierra, S. D. Buckingham, C. L. Campbell, S. Chan, E. Cox, B. R. Evans, T. Fansiri, I. Filipovic, A. Fontaine, A. Gloria-Soria, R. Hall, V. S. Joardar, A. K. Jones, R. G. G. Kay, V. K. Kodali, J. Lee, G. J. Lycett, S. N. Mitchell, J. Muehling, M. R. Murphy, A. D. Omer, F. A. Partridge, P. Peluso, A. P. Aiden, V. Ramasamy, G. Rasic, S. Roy, K. Saavedra-Rodriguez, S. Sharan, A. Sharma, M. L. Smith, J. Turner, A. M. Weakley, Z. Zhao, O. S. Akbari, W. C. t. Black, H. Cao, A. C. Darby, C. A. Hill, J. S. Johnston, T. D. Murphy, A. S. Raikhel, D. B. Sattelle, I. V. Sharakhov, B. J. White, L. Zhao, E. L. Aiden, R. S. Mann, L. Lambrechts, J. R. Powell, M. V. Sharakhova, Z. Tu, H. M. Robertson, C. S. McBride, A. R. Hastie, J. Korlach, D. E. Neafsey, A. M. Phillippy, L. B. Vosshall, Improved reference genome of Aedes aegypti informs arbovirus vector control. Nature 563, 501-507 (2018). 
59. K. Branson, A. A. Robie, J. Bender, P. Perona, M. H. Dickinson, High-throughput ethomics in large groups of Drosophila. Nat Methods 6, 451-457 (2009).

\section{MATERIALS AND METHODS}

\section{Human and animal ethics statement}

Blood feeding of mosquitoes with live mice was conducted according to IACUC protocol 17108. Blood feeding of mosquitoes with human subjects was conducted according to IRB protocol LV-0652. Human subjects gave written informed consent to participate in the experiments.

\section{Mosquito strains}

The following Aedes aegypti strains were used in this paper: wild type Liverpool, Gr3-QF2 (20), Gr4-QF2 (29), fruitless ${ }^{\Delta M}$ (17), fruitless ${ }^{\Delta \mathrm{M}-\mathrm{tdT} T \mathrm{mato}}(17)$, and QUAS-CsChrimson (this paper).

\section{Mosquito rearing}

Mosquito strains were reared at $26^{\circ} \mathrm{C} \pm 2^{\circ} \mathrm{C}$ with $80 \%$ humidity and 14 hours light, 10 hours dark (lights on at 7AM) as previously described (55). Embryos were hatched in hatching broth: 1 pellet of fish food (TetraMin Tropical Tablets, Pet Mountain 16110M) crushed using a mortar and pestle, added to $850 \mathrm{~mL}$ deionized water, then autoclaved. Larvae were reared in deionized water and fed 1-3 tablets of fish food per day. Adult mosquitoes were fed on $10 \%$ sucrose ( $w / v$ in distilled water) ad libitum. Sucrose was delivered in a Boston clear round $60 \mathrm{~mL}$ glass bottle (Fisher FB02911944) filled with $50 \mathrm{~mL} 10 \%$ sucrose. A cotton dental wick (Richmond Dental 201205) was inserted into the bottle and mosquitoes fed from the sugar-moistened wick. Female mosquitoes were blood fed on mice or human arm to generate eggs. Eggs were dried at $26^{\circ} \mathrm{C}$ and $80 \%$ humidity for 3 days, and then stored at ambient temperature and humidity for up to 3 months. Adults were allowed to mate freely for at least 7 days prior to performing experiments. All behavior experiments were carried out in the light phase of the photoperiod, with most experiments occurring between Zeitgeber (ZT) ZT2 to ZT12.

Creation of CsChrimson mosquitoes for optogenetics We generated mosquitoes that expressed a translational fusion of CsChrimson to tdTomato under the control of the QUAS promoter, referred to as CsChrimson or QUASCsChrimson throughout the paper. The coding sequence of CsChrimson-tdTomato was PCR-amplified from the vector p20X (18) using the following oligonucleotide primers: forward 5'- CTCGAGCAAAATGAGCAGACTGGTCGCCGCTTC-3', reverse 5'- ATCCTCTAGATTACACCTCGTTCTCGTAGCAGAATTTATACAG-3'.

The vector backbone from $\mathrm{PXL-Bacll}$ (56) was amplified by PCR using the following oligonucleotide primers: forward 5'GTCTGCTCATTTTGCTCGAGCCGCGGCCGCAGATC-3', reverse 5'-CGAGGTGTAATCTAGAGGATCTTTGTGAAGGAACCTTACTTCTG-3'. The insert was cloned into the backbone using Infusion HD cloning kit (Takara 638920 ) to create pTS26, available at Addgene (plasmid number 175548). This plasmid was injected into 500 Aedes aegypti Liverpool embryos by the Insect Transformation Facility (Rockville, MD) using $200 \mathrm{ng} / \mu \mathrm{L}$ plasmid DNA and 200 $\mathrm{ng} / \mathrm{\mu L}$ piggyBac transposase mRNA. Ten independent QUAS-CsChrimson integration events were isolated under standard mosquito rearing conditions.

\section{Peripheral sensory appendage microscopy}

Mosquitoes 3-4 weeks of age were anesthetized on ice, then maxillary palps and antennae were removed using sharp forceps and placed in fixative (4\% paraformaldehyde, $0.1 \mathrm{M}$ Millonig's Phosphate Buffer pH 7.4, 0.25\% Triton X-100) and nutated for $30 \mathrm{~min}$ at $4^{\circ} \mathrm{C}$. Tissues were washed four times in PBS, then mounted in SlowFade Diamond Antifade Mountant (ThermoFisher). Images were acquired on an Inverted LSM 780 laser scanning confocal microscope (Zeiss) using a 25x 0.8 NA multi-immersion objective with oil. Images were processed using ImageJ.

\section{Brain Immunostaining}

Brain immunostaining was carried out as previously described (29). Mosquitoes 1-2 weeks of age were anesthetized on ice, then heads were removed using forceps and placed into fixative (4\% paraformaldehyde, $0.1 \mathrm{M}$ Millonig's Phosphate Buffer pH 7.4, 0.25\% Triton X-100) and nutated for $3 \mathrm{hr}$ at $4^{\circ} \mathrm{C}$. Heads were washed four times in PBS and kept on ice during dissections. The brains were dissected using \#5 forceps (Dumont) in a droplet of PBS on a Petri dish coated with SYLGARD silicone elastomer (Dow). Brains were transferred to a $35 \mu \mathrm{m}$ mesh cap of a flow cytometry test tube (Fisher 08-771-23) in a 24-well plate containing PBSTx (PBS with $0.25 \%$ Triton X-100). Brains were washed four times for $30-60 \mathrm{~min}$ at room temperature in PBSTx on an orbital shaker before permeabilization and between each of the following steps. Brains were permeabilized in PBS with $4 \%$ Triton X-100 and $2 \%$ normal goat serum for 2 days at $4^{\circ} \mathrm{C}$ on an orbital shaker. We used the mouse anti-Bruchpilot (brp) monoclonal antibody at a dilution of 1:10,000. The brp antibody was purified by Frances Weis-Garcia of the Sloan Kettering Institute Antibody \& Bioresource from the brp/nc82 hybridoma, developed by Erich Buchner at the Universitätsklinikum Würzburg and obtained from the Developmental Studies Hybridoma Bank, created by the NICHD of the NIH and maintained at The University of lowa, Department of Biology, lowa City, IA 52242. Rabbit anti-RFP antibody (Rockland 600-401-379) was used at a dilution of 1:1000 to detect tdTomato fused to CsChrimson. Brains were incubated in primary antibodies in PBSTx with $2 \%$ normal goat serum for 3 days at $4^{\circ} \mathrm{C}$ on an orbital shaker. Secondary antibodies were goat anti-mouse Alexa Fluor 647 (Thermo Fisher A21235) and goat anti-rabbit Alexa Fluor 555 (Thermo Fisher A32732) both at 1:500 dilution. Brains were incubated in secondary antibodies in PBSTx and $2 \%$ normal goat serum for 2 days at $4^{\circ} \mathrm{C}$ on an orbital shaker. Brains were washed four more times at room temperature for 30-60 min before mounting in SlowFade Diamond Antifade Mountant (ThermoFisher). Images were acquired on an Inverted LSM 880 Airyscan NLO laser scanning confocal microscope (Zeiss) using a $25 \times 0.8 \mathrm{NA}$ multi-immersion objective with oil. Images were processed using ImageJ. 


\section{Rearing mosquitoes for optogenetics}

For CsChrimson to respond to red light $(625 \mathrm{~nm}$ in this paper), it is necessary to supply the all-trans retinal co-factor. Moreover, it is critical that animals reared for optogenetics be maintained in the dark to avoid activating CsChrimson inappropriately. Therefore, we developed a mosquito rearing protocol to deliver all-trans retinal under dark conditions. First, we carried out experiments to select the best QUASCsChrimson transgenic insertion among the 10 lines we generated. We wanted lines with strong and selective behavioral induction in combination with a QF2 driver, but no basal behavioral activity without a QF2 driver. We also wanted it to be a single insertion at a known position in the genome, that would not obviously disrupt a known gene. The insertion site of the transgene in each line was mapped to the genome using TagMapping (57). After being fed with all-trans retinal as described below, all 10 lines were tested for their response to red light with and without being crossed to a QF2 driver. Based on these initial screens, a single line with the QUAS-CsChrimson transgene inserted in an intron of the gene LOC23687794 on chromosome 2 at base pair $453,953,698$ in the L5.0 version of the Aedes aegypti genome (58) was selected for use in all subsequent experiments. This CsChrimson strain was outcrossed to wild type mosquitoes for 8 generations before being homozygosed and used for behavior experiments. For all experiments except those in Figure 2I, K (which used wild type mosquitoes with a green light startle stimulus), animals were subjected to special rearing conditions to prepare them for optogenetics. Eggs were hatched in $1 \mathrm{~L}$ of hatching broth under a 14hour $450 \mathrm{~nm}$ blue light and a 10-hour dark cycle in a lighttight $26^{\circ} \mathrm{C}$ incubator with $80 \%$ humidity. Blue light was selected for the light phase of the photoperiod to avoid activating CsChrimson. The next day $2 \mathrm{~L}$ of distilled water was added to the pan, and the following day larvae were thinned to 450 per pan. Larvae were subsequently sorted for fluorescence markers if necessary, using a dissecting microscope. Larvae were fed daily with 1-3 tablets of Tetramin fish food (Pet Mountain 16110M) ground into a powder using a mortar and pestle. Pupae were moved to eclose into adults in a $30 \mathrm{~cm} \times 30 \mathrm{~cm} \times 30 \mathrm{~cm}$ insect rearing cage (Bugdorm DP1000) with ad libitum access to $10 \%$ sucrose in sugarfeeding glass bottles. Animals were not sexed at this stage, so cages contained males and females that freely mated. Behavior experiments were performed 1-4 weeks post-pupation. Three days before the experiment, the sugar wicks were replaced with water wicks to starve animals for 24 hours. Two days before the experiment, the water wicks were replaced with wicks soaked in $10 \%$ sucrose and 400 $\mu \mathrm{M}$ all-trans retinal (Sigma, R2500-1G). $50 \mathrm{~mL}$ of sucrose and all-trans retinal was used per cage. Animals were allowed to feed for 1-3 days in the dark on this meal. In pilot experiments we verified that starved females fed on sucrose and all-trans retinal by observing yellow pigmentation in the abdomen. Feeding in the dark was used to avoid premature neuronal activation and bleaching of the all-trans retinal in the sugar feeders. This rearing protocol was used for all experiments in the opto-membrane feeder, opto-thermocycler, and opto-feeder experiments.

\section{Opto-membrane feeder assay}

The optomembrane feeder assay was constructed using optomechanical components (Thorlabs MB12, TR12, RA90) and a black $1 / 4$ " thick acrylic platform for the canister of mosquitoes to rest on. A hole in the bottom of the platform allowed a camera (Blackfly U3-13S2M-CS, FLIR) outfitted with an $800 \mathrm{~nm}$ longpass filter (Midwest Optical LP800-34) to image through the clear canister. Canisters were constructed from a polycarbonate tube of diameter 4.5" (McMaster-Carr 8585K56) and 5" long. The bottom was made of clear $1 / 8$ " thick acrylic and attached with plastic epoxy (Loctite 1363118). The top was an inset lid made of black 1/4" and 1/8" inch acrylic and UV-resistant black mesh (McMaster-Carr 87655K13). The canister was surrounded by a coil of RGB LEDs (Digikey 289-1189-ND) spaced 1.5" from the exterior of the canister and controlled by an Arduino Uno board (Arduino A000066). Mosquitoes were illuminated by $850 \mathrm{~nm}$ infrared LEDs surrounding the top of the cylinder of RGB LEDs. The assay was enclosed in a black $1 / 4$ " thick acrylic box of dimensions 15 " x 15 " x 28 " to prevent ambient light from entering the assay. The top of the acrylic box had an entry port of 4" x 2.7" for $\mathrm{CO}_{2}$ diffused by a Flystuff Flypad (Genesee Scientific, 59-114), and two doors on the side, one at the level of the cylinder (10" high $\times 8$ " wide) and one at the bottom (8" high $\times 10$ " wide) at the level of the camera. The day before the experiment, mosquitoes were sexed under cold anesthesia in white light, placed into the cylindrical canisters, and fed water and $400 \mu \mathrm{M}$ all-trans retinal in the dark until the experiment commenced. Dental wicks were soaked in approximately $12.5 \mathrm{~mL}$ of the water and all-trans retinal, placed on top of the mesh of the inset lid. Trials were run in an environmental room at $25-28^{\circ} \mathrm{C}$ and $70-80 \%$ humidity. For each trial, a canister of 20 mosquitoes was placed on the platform and acclimated for $10 \mathrm{~min}$ prior to the stimulus. Throughout the acclimation period and trial, the canister was bathed in dim blue $471 \mathrm{~nm}$ light from the RGB LEDs. At the start of the trial, a blood meal consisting of 5 $\mathrm{mL}$ of defibrinated sheep blood (Hemostat Laboratories DSB100) with 2 mM ATP (Sigma A6419-1G) heated to $42^{\circ} \mathrm{C}$ was placed on top of the canister. Blood meals were delivered using an acrylic lid consisting of a 1/16" thick clear acrylic ring with a 2" inner diameter and 2.6" outer diameter attached to a $1 / 2$ " thick clear acrylic ring with a 2.3 " inner diameter and 2.6" outer diameter. This lid was covered with Parafilm on the $1 / 16$ " thick side to create a well for the blood when placed Parafilm-down on the inset lid of the cylindrical canister. At the start of the experiment, the warm blood meal was pipetted onto the Parafilm. On top of the blood meal was an inverted 4 oz bottle (SKS Bottle \& Packaging 060407) filled with water heated to $42^{\circ} \mathrm{C}$ to keep the blood near body temperature for the duration of the $15 \mathrm{~min}$ trial. Mosquitoes were given $\mathrm{CO}_{2}$, red light $\left(624 \mathrm{~nm}, 3.5-6 \mu \mathrm{W} / \mathrm{mm}^{2}\right)$, or neither stimulus throughout the $15 \mathrm{~min}$ trial. $10 \% \mathrm{CO}_{2}$ was mixed with filtered room air using flow controllers (Aalborg P26A1-BA2) to deliver a $2.7 \% \mathrm{CO}_{2}$ stimulus through the top of the container. Air flow delivery is described in detail in (17). Between trials, the lower door was opened for $5 \mathrm{~min}$ with the air flow on to flush residual $\mathrm{CO}_{2}$ from the assay On a given day of experiments, each of 3 genotypes (Gr3, CsChrimson, and Gr3 > CsChrimson) was tested with each 
of the 3 stimuli (no stimulus, $\mathrm{CO}_{2}$, red light), for a total of 9 trials. The order of trials was rotated between days. Genotypes were blinded to the experimenter. Attraction to the warm blood meal was quantified by manually counting the number of mosquitoes on the warm blood meal in the video ( 1 frame/sec) every $15 \mathrm{sec}$. Engorgement was quantified by visual examination of mosquitoes at $4^{\circ} \mathrm{C}$ after the end of the trial. Between days of experiments, the canisters were cleaned by spraying $70 \%$ ethanol with a spray bottle and wiping down with a soft sponge, rinsed with deionized water, and air dried.

\section{Opto-thermocycler assay}

The opto-thermocycler assay was constructed on top of a PCR thermocycler (Eppendorf Mastercycler) using optomechanical components (Thorlabs XE25L12, XE25L24, XE25L09, XE25T4, RA90, TRA6, TR12). This assay was used as the basis for experiments delivering light only, light along with heat stimuli, and opto-feeder experiments. Light was delivered using six red light 627nm LEDs (Luxeon Star SP-01-D9) or six green light 530nm LEDs (Luxeon Star SP01-G4) controlled with an Arduino Uno board. Light intensity was measured using a Coherent Wand UVIVIS Power Sensor (1299161). The surface of the PCR block was covered in black tape to reduce glare (Thorlabs T137-2.0). Temperature was measured using a type $T$ thermocouple (Harold G. Schaevitz Industries LLC CPTC-120-X-N) connected to the Arduino board (Arduino A000066) using a thermocouple amplifier (Adafruit MAX31856). The thermocouple sensor was placed on the surface of the lower right of the PCR block and secured using black tape (Thorlabs T137-2.0). Temperature reading and light output were recorded every 100 msec from the Arduino using a custom Processing script. Video was synchronized with the light and temperature stimuli with an infrared $940 \mathrm{~nm}$ LED (Adafruit 387) covered with tape and placed in the field of view of the camera. Mosquitoes were illuminated with an infrared $850 \mathrm{~nm}$ LED strip (Waveform Lighting 7031.85) surrounding the plate of mosquitoes orthogonal to the view of the camera. Video was recorded using a Blackfly camera (FLIR BFS-U3-16S2M-CS) outfitted with a $780 \mathrm{~nm}$ longpass filter (Vision Light Tech LP780-25.5) at 30 frames/sec using Spinview software. Heat stimuli were programmed onto the PCR thermocycler to elicit the desired change in temperature from ambient to skin temperature $\left(25-35^{\circ} \mathrm{C}\right)$ as measured by the thermocouple (Figure 2B). Light stimuli were $627 \mathrm{~nm}$ at an intensity of $12 \mu \mathrm{W} / \mathrm{mm}^{2}$. To synchronize the heat and light stimuli, experiments started with a brief dip in temperature followed by a 10 min acclimation period after which the experiment started. Experiments in Figure $2 \mathrm{E}-\mathrm{H}$ were conducted with a single stimulus presented to mosquitoes to determine the duration of response. In all other experiments, mosquitoes received multiple stimuli over the course of a 3-6 hr experiment. Data from the rare trials where the mosquito died in the course of the experiment were discarded. For experiments using red light and heat, trials were delivered $20 \mathrm{~min}$ apart and the order was pseudorandomized between multiple sweeps of trials and across days. For experiments using green light and heat (Figure $21, K$ ), the stimuli were pseudorandomized across sweeps only. Mosquitoes were assayed in a custom acrylic plate with $3 \times 5$ wells. The sides of the plate were cut using a laser cutter from 1/8" thick clear acrylic, then assembled using acrylic glue (WELD-ON, \#4SC Plastic Solvent Glue for Acrylic). The top and bottom were cut from $1 / 16$ " acrylic. The top was left removable to load mosquitoes while the bottom was used to sandwich a piece of black fiberglass window screen (Breakthrough Premium Products IHLRS3684BL) creating a mesh bottom for each well. The acrylic bottom piece spaced the mesh bottom of the wells $1.5 \mathrm{~mm}$ from the surface of the PCR block. Wells containing the mosquitoes were $18.5 \mathrm{~mm}$ long $\times 17 \mathrm{~mm}$ wide $x 12 \mathrm{~mm}$ high. The well in the lower right was empty to accommodate the thermocouple. The day before the experiment, mosquitoes were sexed under cold anesthesia in white light, placed into the custom plate, and fed water and $400 \mu \mathrm{M}$ all-trans retinal overnight until the experiment. This was delivered in cotton dental wicks each soaked with 12.5 $\mathrm{mL}$ water and all-trans retinal. Three wicks were laid flat beneath each plate so that mosquitoes in all wells could access the wicks beneath. Experiments were run at ambient room temperature and humidity, but the PCR block kept the assay chamber at a fixed temperature. Between trials the surface of the PCR block was cleaned by wiping with a Kimwipe moistened with $70 \%$ ethanol. Between days of experiments, the canisters were cleaned by spraying $70 \%$ ethanol with a spray bottle and wiping down with a gloved finger, rinsed with deionized water, and air dried.

\section{Opto-feeder assay}

The opto-thermocycler assay captures probing behavior but does not offer a meal for engorgement. We therefore modified this device to produce the opto-feeder. The most biologically relevant meal for host-seeking females would be blood, but its opacity makes it unsuitable for our video tracking. We therefore used adenosine $5^{\prime}$-triphosphate (ATP) in saline as a an optically clear proxy for blood. This meal has previously been shown to be highly palatable and triggers mosquito engorgement equivalent to a blood meal $(29,34)$. To modify the opto-thermocycler to accommodate this blood meal substitute, a thin aluminum plate (McMaster-Carr 6061 Aluminum sheet 0.025 ") was sandwiched between laser cut pieces of acrylic creating wells on the side facing the mosquito. The wells were $18.5 \times 17 \times 1 \mathrm{~mm}$. The acrylic was bonded to itself using acrylic glue (WELD-ON, \#4SC Plastic Solvent Glue for Acrylic) and to the aluminum plate with epoxy (Loctite 1363118) and UV-curing glue (Bondic SK8024). The plate was prepared for a trial by adding 500 $\mu \mathrm{L}$ of the meal $\left(110 \mathrm{mM} \mathrm{NaCl}, 20 \mathrm{mM} \mathrm{NaHCO}_{3}\right.$, and $2 \mathrm{mM}$ ATP) to each well of the plate in Figure 4A-C. In Figure 4D$\mathrm{F}$, the composition of the meal was $110 \mathrm{mM} \mathrm{NaCl}, 10 \mathrm{mM}$ $\mathrm{NaHCO}_{3}$, and $2 \mathrm{mM}$ ATP. The plate was covered with Parafilm to provide a membrane for the mosquitoes to pierce before accessing the meal. The plate was placed directly on top of the PCR bock to allow maximum heat transfer. The thermocouple was placed on the surface of the Parafilm in the middle of the well in the lower right corner to record the temperature of the heated meal. Trials were carried out and synchronized in the same way as opto-thermocycler experiments. All opto-feeder experiments were single trial. 
Machine-learning based behavior classification

Videos were pre-processed using a custom Python script tracking_optothermo.py that converted the file format, split up videos into $\sim 30$ min chunks, selected frames to create a background image for centroid tracking, and detected frames where the IR synchronization LED was illuminated. Next, we used Ctrax (59) for centroid tracking. A background model was created using the selected frames from the experimental video. Ctrax background settings were background brightness high threshold 2.55, low threshold 0.250.5 adjusted depending on the video. The area with the infrared synchronization LED was excluded using a region of interest to avoid interference with the tracking. Mosquitoes that moved very little, such that they were visible in the background image, were corrected for using the Fix Background Model option. In tracking settings, shapes were filtered using the following minimum/maximum: 110/1600 for area, 4/36 for major axis, 4/30 for minor axis, 0.0/0.98 for eccentricity. The rest of the tracking settings were default. We used Ctrax centroid tracking as input to Animal Part Tracker (APT, https://github.com/kristinbranson/APT downloaded on July $9,2020)$ for tracking points on the mosquito body. For optothermocycler experiments we tracked 9 points: the tip and base of the proboscis, the tip of the abdomen, and 3 points on each foreleg: where the femur connects to the body, the join between the femur and the tibia, and the joint between the tibia and the first tarsomere. Opto-thermocycler classifiers were trained on 320 frames from two videos for female mosquitoes and 102 frames from one video for male mosquitoes. We tracked 13 points for opto-feeder experiments, the same 9 points as for opto-thermocycler experiments plus two points at the point of the abdomen where it connects to the thorax and two points at the midpoint or thickest part of the abdomen. The opto-feeder classifier was trained on 215 frames from four videos. All APT classifiers were trained using the Cascaded Pose Regression tracking algorithm. Janelia Automatic Animal Behavior Annotator [(JAABA) (22), downloaded on July 15 2020] was used for classifying specific behaviors. The classifier for flight (called fly2) was used for all videos of females and males. It was trained from two videos and used appearance and locomotion features with radius of 10 frames with no post-processing. The other classifiers additionally used APT information, a larger radius of frames, and minimum bout sizes for improved accuracy. Separate classifiers were trained for females and males in the opto-thermocycler and females in the opto-feeder experiments to maximize classifier accuracy in the face of differences in visual appearance. Probing classifiers (probe5 for female opto-thermocycler experiments, probemale for male opto-thermocycler experiments, and probeBB for females in the opto-feeder experiments) included the pair of points proboscis tip and base as features, along with APT, motion, and appearance features. The grooming and walking classifiers (walk3 for female and male opto-thermocycler experiments, groom3 and groommale for female and male opto-thermocycler experiments, respectively, and walkBB and groomBB for opto-feeder experiments) were trained using APT, locomotor, and appearance features. APT classifiers were visually inspected for accuracy. APT and JAABA classifiers were evaluated by the accuracy of ground truthing on the JAABA classifiers. An initial classifier was trained, then ground truthing was performed on 50-100 segments of $1 \mathrm{sec}$ video segments that were balanced between segments with and without the behavior. These segments were examined for misclassified frames and additional training was performed to improve the classifier. Thus, the ground truth dataset is more challenging than a random one because it contains frames that were previously mis-classified and so the real accuracy is higher. Training continued until true positive and true negative rates of were $>90 \%$ were obtained with 7 of 9 classifiers. Two other classifiers had rates slightly below this. The groomBB was trained to $\sim 84 \%$ true positive and negative rate because certain grooming postures are difficult to distinguish from probing postures. The probemale classifier was trained to $\sim 87 \%$ true positive and $\sim 91 \%$ true negative rate because only part of the male proboscis is distinguishable from the maxillary palps during probing behavior. Classified behaviors for each mosquito track from JAABA were assigned to single wells according to $x-y$ location of the track to correct the small numbers of frames where Ctrax detected two mosquitoes per well (usually due to a leg that was discontinuous with the rest of the animal) and to connect broken tracks to a single individual mosquito. The IR LED stimulus in the video was aligned with data about temperature and light stimuli from the Arduino and assigned to frames in the video. Velocity was calculated by taking the Ctrax $x-y$ position at $100 \mathrm{~ms}$ intervals ( 3 frames).

\section{Analysis of behavior}

To calculate the half-life of the mosquito behavior response in Figure $2 \mathrm{H}-\mathrm{J}$ and Figure $3 \mathrm{C}, \mathrm{H}$, the baseline was calculated as the average probing in 2 min prior to stimulus onset. A sliding window of the amount of probing was calculated in $15 \mathrm{sec}$ windows starting at stimulus onset for every frame. The maximum response was defined as the window with the greatest probing after stimulus onset and $t_{1 / 2}$ was defined as the first window in which the probing was halfway between the maximum response and the baseline. To calculate the integration of heat and the second stimuli (fictive $\mathrm{CO}_{2}$, fictive sugar, or green light) in Figure S2 and Figure 2M, we calculated the average response to each of the individual stimuli. We added the two responses to get a predicted additive response. For each individual mosquito, we divided its response by the predicted additive response and multiplied by $100 \%$. This gave a percent additivity where $0 \%$ was no response and $100 \%$ was exactly additive. For line graphs, the additivity signal was smoothed over $4.5 \mathrm{sec}$ around each 500-millisecond timepoint.

\section{tSNE analysis}

To infer the state of individual mosquitoes in the opto-feeder experiment, we split each mosquito track into $30 \mathrm{sec}$ intervals at 10 sec step size and calculated 38 parameters. The $30 \mathrm{sec}$ time interval was selected as a period of time over which the behaviors exhibited were relevant to interpreting the internal state of the mosquito. The time interval was varied from 10 to $60 \mathrm{sec}$ to assure that the results were not sensitive to this parameter choice (Figure S4). The parameters included the proportion of the time window that mosquitoes exhibited each behavior and no behavior. Mosquitoes 
can probe and walk at the same time so the proportion of time probing and walking, probing not walking, and walking not probing were included. The number of bouts of each behavior was included. Velocity parameters included average velocity over the window and average velocity during each behavior. Transitions between behaviors were included as outgoing rate per second of transition to all other behaviors or no behavior. For the purposes of transitions and ethograms, probing and walking were treated as mutually exclusive with probing taking higher precedence over walking. For all behaviors to avoid rare frames where multiple behaviors were classified for a single frame the precedence of behaviors were flying $>$ probing $>$ walking $>$ grooming $>$ no behavior. Based on the total amount of time animals spent performing each behavior, cutoffs were determined to specify a minimum amount of behavior exhibited. Cutoffs were 0.04 for flight, 0.2 for walking or probing, and 0.3 for grooming. Behavior below these cutoffs was excluded from further analysis. The Python package scikit-learn (https://scikit-learn.org/ version 0.24.1) was used for tSNE embedding with parameters $n / 100$ perplexity (1061), and other default parameters (200 learning rate, 1000 iterations). Multiple perplexities were compared to assure that results were not sensitive to this parameter choice. tSNE plots were examined and clusters were segmented manually by grouping densely clustered points. These clusters were used to annotate videos for visual inspection of what mosquito behaviors they corresponded to. Names for clusters were chosen based on the characteristics of the clusters shown in Figure 4K, Figure S3B, Figure S4, and video observation. Clusters that included mosquitoes that moved around were named Global or Local search based on the total amount of movement, and contrasting amounts of flight and walk behaviors. The cluster that included mostly grooming was termed Rest. The cluster that included mosquitoes that were stationary, probing, and with abdomens expanded after feeding was termed Engorge. The clusters for Rest, Global Search, and Local Search were single clusters that were clearly differentiated on the tSNE embedding. The Engorge cluster was composed of two smaller clusters that, when observed on video, both consisted of mosquitoes engorging and were therefore combined. Points on the end of the Local Search cluster in the tSNE embedding with high probing were also examined by video and grouping was kept with the Local Search cluster.

\section{Statistical analyses}

R (https://www.r-project.org version 4.0.5) and Python were used for statistical analysis. Data distributions were visually examined for normality or tested using the Shapiro-Wilk test. Normally distributed samples were compared by one sample t-test for paired measurements or ANOVA and Tukey's test for multiple categories. Non-normally distributed samples were compared using the Friedman test for multiple category repeated measurements, Kruskal-Wallis test for multiple category single measurements, or the sign test for skewed paired measurements. The Friedman and KruskalWallis test were used with Nemenyi post-hoc tests to determine pairwise differences between categories. T-tests and sign tests were adjusted using Holm's method for correcting for multiple comparisons. For statistical analyses involving comparisons of the behavior of males and females, we repeated the tests after accounting for differences in classifier accuracy by changing the proportion of behavior by this difference (i.e. $4.93 \%$ for probing and $3.25 \%$ for walking) and confirming that the results were the same. Sample sizes followed conventions in the field. For experiments with multiple stimuli presented to each animal, 4-6 days of data were collected. For endpoint and single stimulus experiments, 7-11 days of data were collected.

\section{Logistic Regression}

Logistic regression models for the opto-feeder experiment were trained using the Python sklearn package with the proportion of time mosquitoes spent in each of the four behaviors (groom, walk, probe, and fly) for two minutes after the light stimulus as predictors. These periods of time were -2 to 0 minutes, -8 to -6 minutes, and -14 to -12 minutes relative to the heat stimulus for the 2 minute, 8 minute, and $14 \mathrm{mi}-$ nute inter stimulus interval experiments. The dependent variable was whether the mosquito engorged by the end of the experiment. Models used the liblinear solver, random_state of 0 , and balanced weight_class. Bootstrapping was performed using 10,000 resamples with replacement of the engorgement dataset to determine the distribution of predictive models. 10,000 shuffles of the engorgement data were used to determine whether the predictive model performed above chance. Leave-one-out cross-validation was used to determine whether the model was overfitted.

\section{Code availability}

Analysis code used in this publication is available at github.com/trevorsorrells/Optothermocycler. 


\section{SUPPLEMENTARY FIGURES}
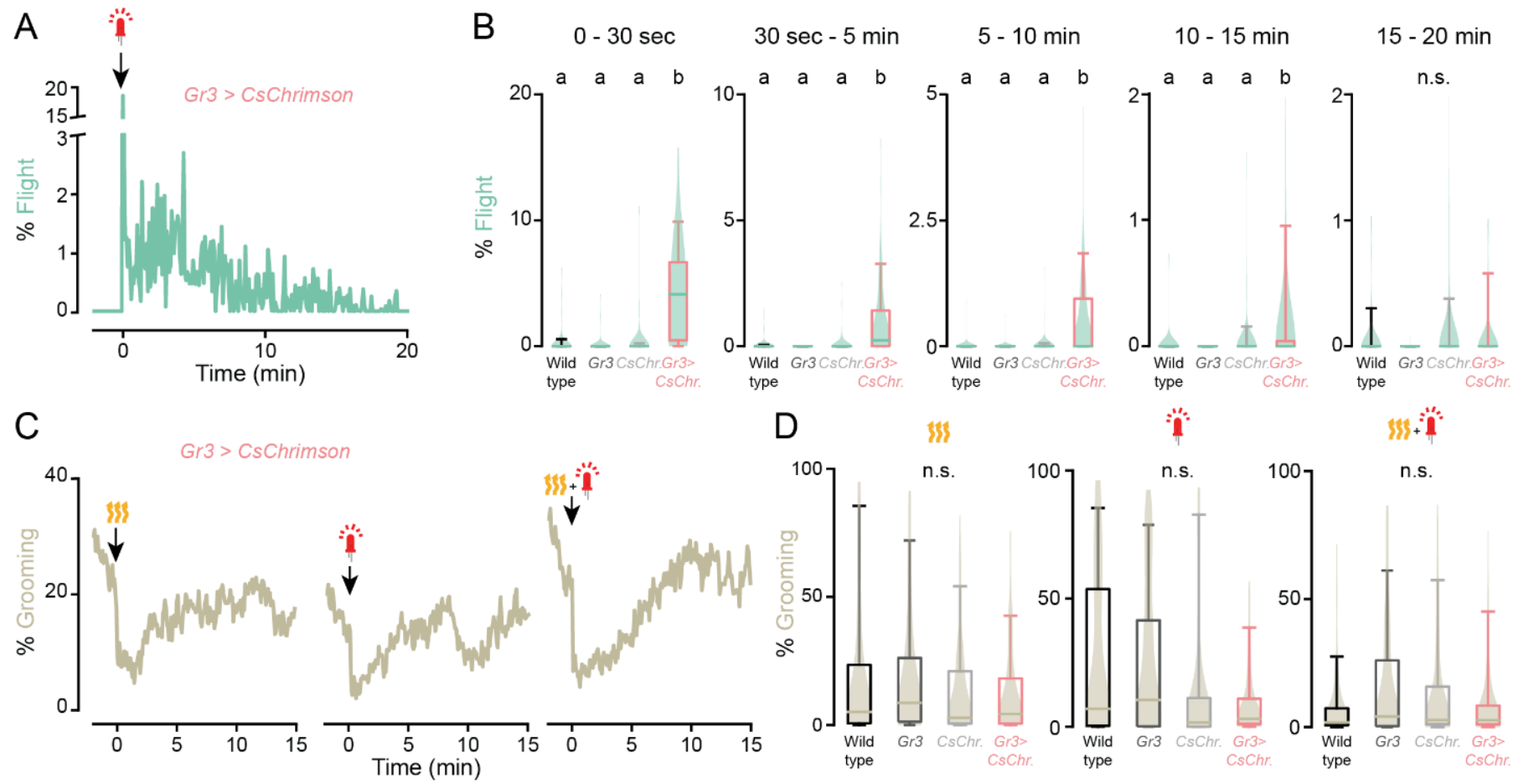

Figure S1. Fictive $\mathrm{CO}_{2}$ triggers flight events for 15 minutes (Related to Figure 2)

(A, C) Percent indicated behavior of Gr3 > CsChrimson females from 2 min before to 15 min after stimulus onset in response to the indicated stimuli. Data from Figure 2E-H. (B, D) Quantification of the behavior in A, C along with data collected from the additional indicated genotypes. Data from Figure 2E-H. Data are plotted as violin-box plots (median: horizontal line, interquartile range: box, 5th and 95th percentiles: whiskers). Data labeled with different letters are significantly different $(P<0.05$, Kruskal-Wallis test followed by Nemenyi post-hoc tests. n.s., not significant, $n=68-70$ mosquitoes). 
bioRxiv preprint doi: https://doi.org/10.1101/2021.10.06.463436; this version posted October 7, 2021. The copyright holder for this preprint (which was not certified by peer review) is the author/funder, who has granted bioRxiv a license to display the preprint in perpetuity. It is made available under aCC-BY 4.0 International license.

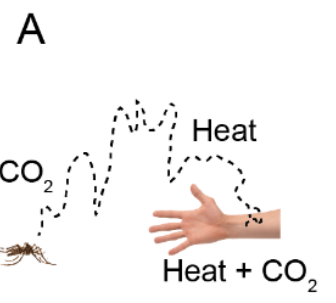

B Gr3 > CsChrimson

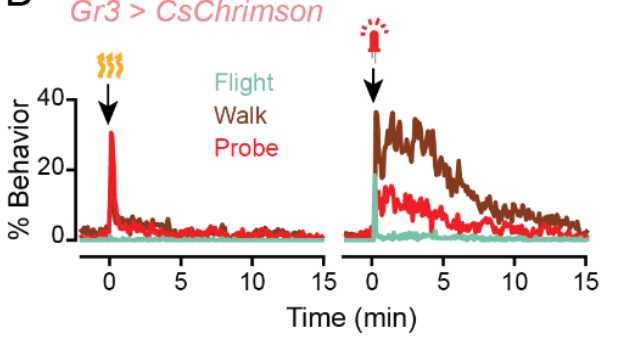

$\mathrm{E}$

Gr3 > CsChrimson $313+\because "=$

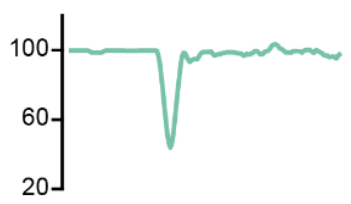

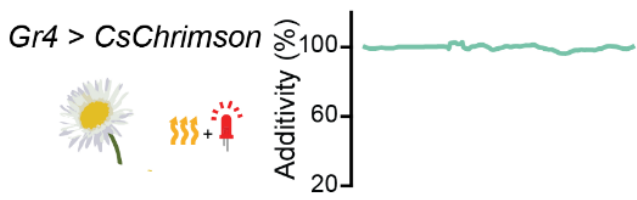

Wild type

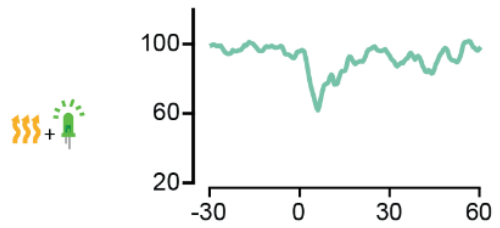

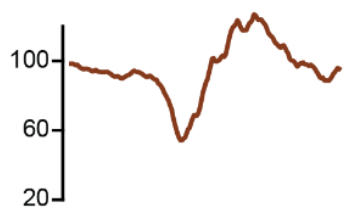
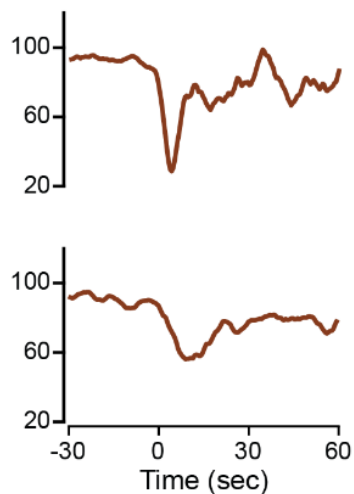
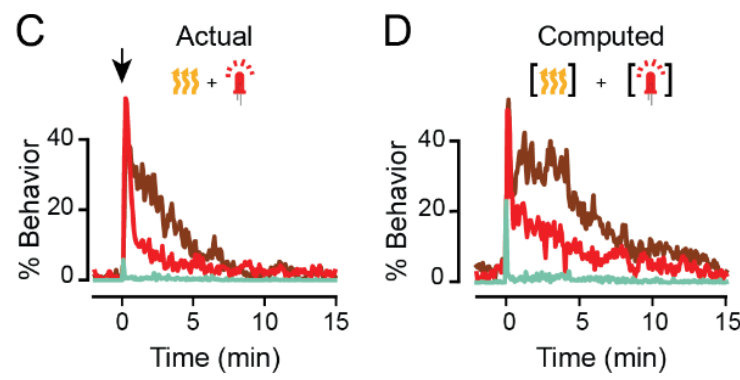

$\mathrm{F}$
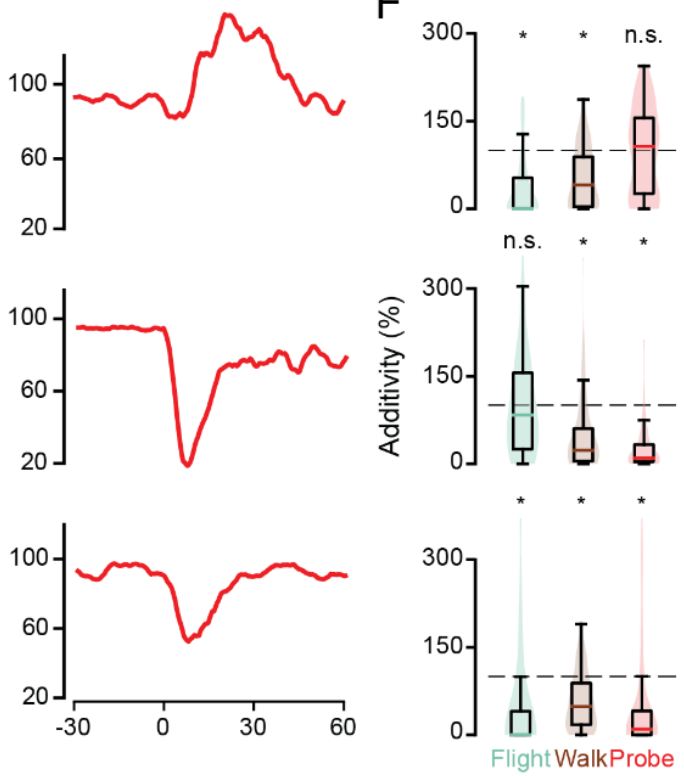

Figure S2. Host cues are integrated with different computations than non-host cues (Related to Figure 2)

(A) Mosquitoes sense host cues separately and simultaneously at different distances from the host. (B-D) Responses to individual (B) or combined (C) stimuli or a computed sum of individual stimuli in B (D). Plots in B-C are reprinted from Figure $2 \mathrm{H}$ for comparison with (D). (E) Differences between calculated and actual responses for the indicated genotypes and stimuli from 30 sec before to 1 min after stimulus onset, smoothed using a box filter of radius 2.25 sec. $n=70$ for Gr3 > CsChrimson, $n=69$ for Gr4 > CsChrimson, $n=140$ for wild type. (F) Quantification of E for the first $15 \mathrm{sec}$ after stimulus onset. Data are plotted as violin-box plots (median: horizontal line, interquartile range: box, 5th and 95th percentiles: whiskers. ( ${ }^{\star} P<0.0001$, sign test using Holm's correction for multiple comparisons, n.s., not significant). 
bioRxiv preprint doi: https://doi.org/10.1101/2021.10.06.463436; this version posted October 7, 2021. The copyright holder for this preprint (which was not certified by peer review) is the author/funder, who has granted bioRxiv a license to display the preprint in perpetuity. It is made available under aCC-BY 4.0 International license.

A
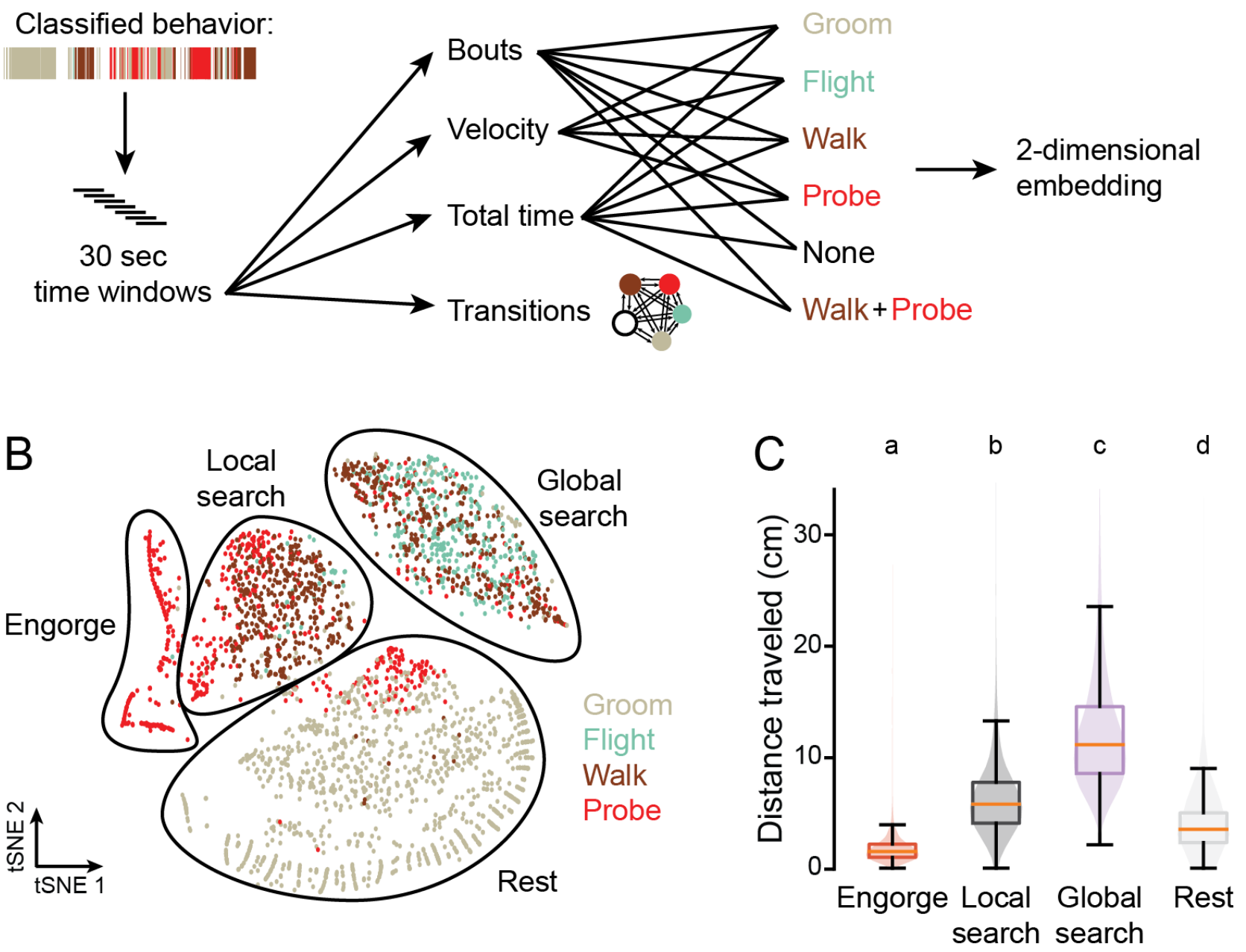

Figure S3 Inference of internal state from behavior (Related to Figure 4)

(A) Schematic of analysis. Classified behavior reflects the output of APT followed by JAABA behavior classifiers. (B) t-stochastic neighbor embedding (tSNE) for $30 \mathrm{sec}$ time windows spanning 4 min prior to the light stimulus to 10 min after heat onset for individual mosquitoes from Figure 4J. Points are colored by strong enrichment of the corresponding behavior. State categories were bounded with a black line by visual inspection of the tSNE plot, graphing the behavior characteristics of each cluster, and comparing with video of the mosquitoes in each state. Shown is a random subset of 3,000 time windows from 106,076 total windows from all 7 stimulus types, n=1,162 mosquitoes. (C) Total distance traveled over the course of $30 \mathrm{sec}$ time windows for each behavior state with the following number windows from each state: engorge, $n=5,634$; local search, $n=11,124$; global search, $n=15,466$; rest, $n=9,891$. Time windows were taken from 4 min before the light stimulus to $10 \mathrm{~min}$ after heat onset. Data are plotted as violin-box plots (median: horizontal line, interquartile range: box, 5th and 95th percentiles: whiskers). Data labeled with different letters are significantly different $(P<0.0001$, Kruskal-Wallis test followed by Nemenyi post-hoc tests). 
bioRxiv preprint doi: https://doi org/10.1101/2021.10.06 463436; this version posted October 7, 2021. The copyright holder for this preprint (which was not certified by peer review) is the author/funder, who has granted bioRxiv a license to display the preprint in perpetuity. It is made available under aCC-BY 4.0 International license.

A

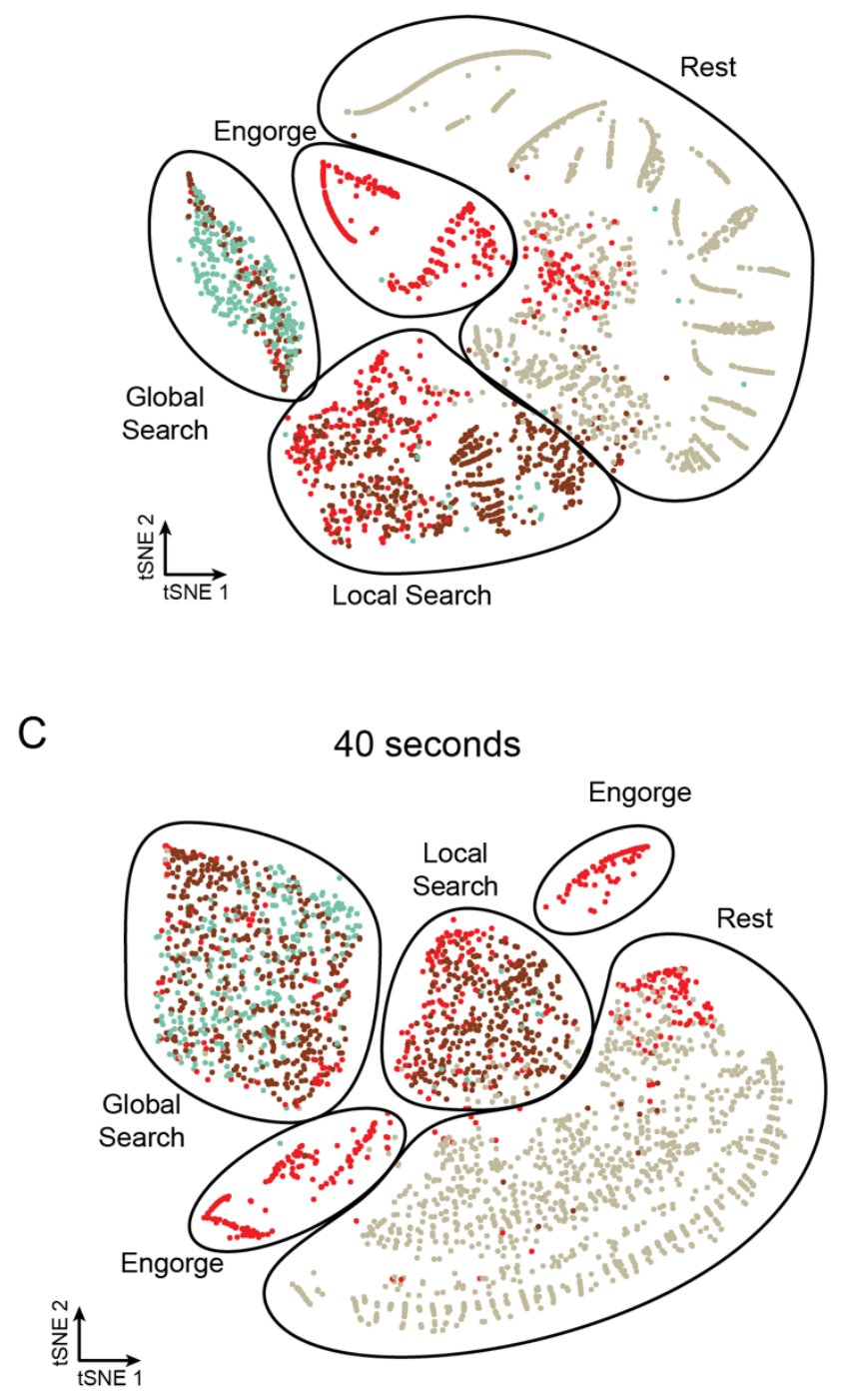

B

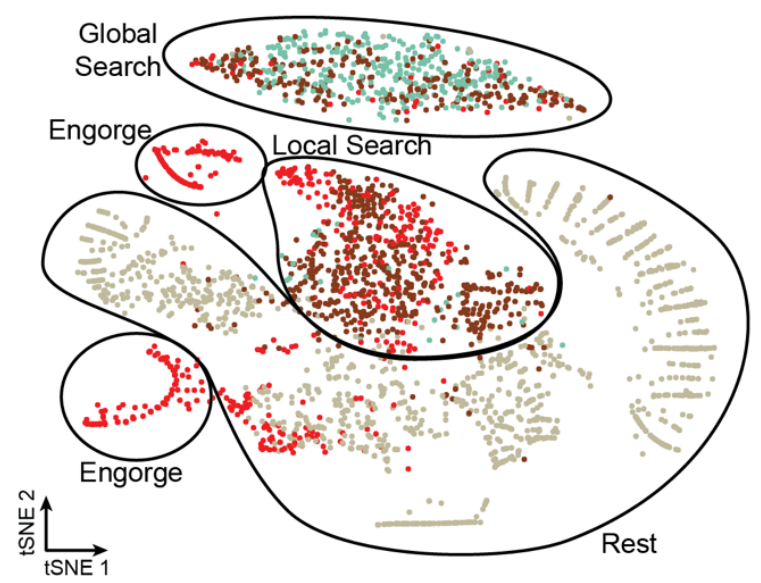

D

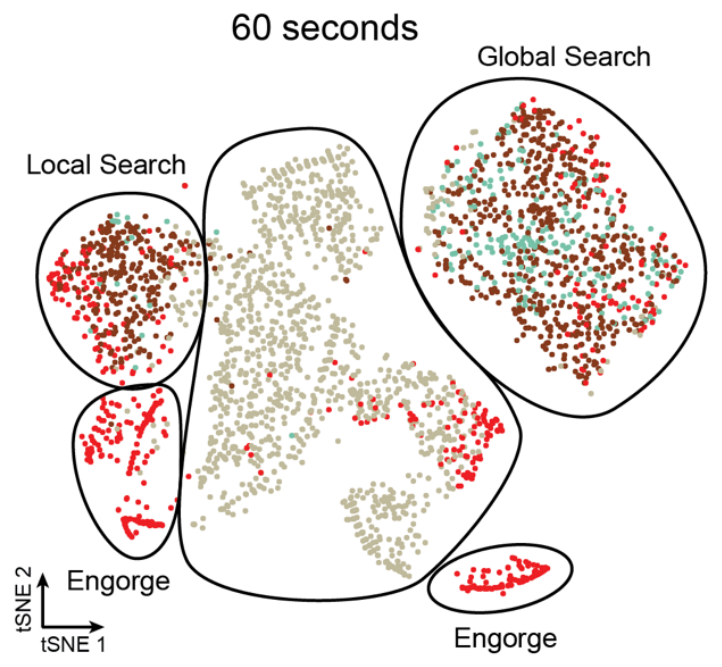

Figure S4. Mosquito states are consistent across window duration. (Related to Figure 4)

(A-D) tSNE for time windows of the indicated duration for individual mosquitoes from. Points are colored by strong enrichment of the corresponding behavior. State categories were bounded with a black line by visual inspection of the tSNE plot. Shown is a random subset of 3,000 time windows from (A) 103,658, (B) 105,387, (C) 106,044, and (D) 105,287 total windows from all 7 stimulus types, $n=1,162$ mosquitoes. 